\title{
Rethinking Defeasible Reasoning: A Scalable Approach
}

\author{
MICHAEL J. MAHER \\ Reasoning Research Institute, Australia \\ (e-mail: michael.maher@reasoning.org.au)
ILIAS TACHMAZIDIS, GRIGORIS ANTONIOU, STEPHEN WADE
University of Huddersfield, $U K$
(e-mail: $\{$ i.tachmazidis, g.antoniou, s.j.wade\}@hud.ac.uk) \\ LONG CHENG \\ Dublin City University, Ireland \\ (e-mail: long. cheng@dcu.ie) \\ submitted 1 January 2003; revised 1 January 2003; accepted 1 January 2003
}

\begin{abstract}
Recent technological advances have led to unprecedented amounts of generated data that originate from the Web, sensor networks and social media. Analytics in terms of defeasible reasoning - for example for decision making - could provide richer knowledge of the underlying domain. Traditionally, defeasible reasoning has focused on complex knowledge structures over small to medium amounts of data, but recent research efforts have attempted to parallelize the reasoning process over theories with large numbers of facts. Such work has shown that traditional defeasible logics come with overheads that limit scalability. In this work, we design a new logic for defeasible reasoning, thus ensuring scalability by design. We establish several properties of the logic, including its relation to existing defeasible logics. Our experimental results indicate that our approach is indeed scalable and defeasible reasoning can be applied to billions of facts.

This paper is under consideration in Theory and Practice of Logic Programming (TPLP).
\end{abstract}

KEYWORDS: Defeasible Reasoning, Parallel Reasoning, Scalability

\section{Introduction}

Recent technological advances have led to unprecedented amounts of generated data that originate from the Web, sensor networks and social media. Once this data is stored, the challenge becomes developing solutions for efficient processing of the vast amounts of data in order to extract additional value. Analytics in terms of reasoning - for example, for decision making should be performed using rule sets that would allow the aggregation, visualization, understanding and interpretation of given datasets and their interconnections. Specifically, one should use rules able to encode inference semantics, as well as commonsense and practical conclusions in order to infer new and useful knowledge based on the data.

Various monotonic logics have been implemented with this large scale of data in mind. Work includes Datalog (Leone et al. 2019, Condie et al. 2018; Martinez-Angeles et al. 2013), $\mathcal{E L}^{+}$ (Mutharaju et al. 2015), OWL Horst (Kim and Park 2015; Urbani et al. 2012), RDFS (Heino 
and Pan 2012; Goodman et al. 2011; Oren et al. 2009) and Fuzzy logics (Zhou et al. 2013, Liu et al. 2011), scaling reasoning up to billions of facts. For a comprehensive overview of existing approaches on large-scale reasoning, readers are referred to (Antoniou et al. 2018).

Nevertheless, it should be pointed out that available data often come from heterogeneous sources that are not necessarily controlled by the data engineer, and therefore may contain imperfect, incomplete or conflicting information. Other reasons for imperfect data may be faults in sensors or the communication infrastructure. It is evident that monotonic reasoning is not suited for such data processing, which subsequently led to the study of large-scale nonmonotonic reasoning. In particular, logic programs under the well-founded semantics (Gelder et al. 1991) has been addressed (Tachmazidis and Antoniou 2013: Tachmazidis et al. 2014). However, this semantics can only indirectly address conflicting information.

Defeasible reasoning provides facilities to directly address conflicting information. While computationally simple, it has found numerous applications in the modelling of legal reasoning (Prakken 1997; Governatori and Maher 2017), regulations (Antoniou et al. 1999; Islam and Governatori 2018), business rules (Grosof et al. 1999), contracts (Governatori and Pham 2009), negotiation (Skylogiannis et al. 2007) and business process compliance management (Governatori et al. 2006, Hashmi et al. 2018).

However, its scalability is still in question. Propositional defeasible logics can be executed in linear time (Maher 2001; Billington et al. 2010) but that algorithm does not easily support parallelism, nor does it easily extend to first-order defeasible logics. Implementations of defeasible logic for big data have been limited to subsets of the full logic (Tachmazidis et al. 2012, Tachmazidis et al. 2012). Both approaches have been applied to billions of facts, but neither approach was able to capture the general case. A fundamental problem in existing defeasible logics identified in (Tachmazidis et al. 2012), is that the notion of provable-failure-to-prove, which is central to these logics, requires the generation and retention of a prohibitive amount of negative derivation conclusions. This inhibits the scalability of implementations of such logics.

In this work, we propose a novel approach for defeasible reasoning over large data by defining a scalable defeasible logic. The new inference rules of the logic avoid reliance on provablefailure-to-prove by building solely on definitely and defeasibly provable conclusions. With this approach, the new logic provides scalability by design. The result is a reasoning process that is comparable in terms of scalability with existing methods for monotonic logics. In the context of this work, a scalable method allows large-scale inference computation by utilizing parallel and distributed settings over big data. Experimental results highlight the scalability properties of the proposed logic, while showing that our approach can scale up to 1 billion facts over a real-world case study.

The paper is structured as follows. Section 2 provides a brief outline of defeasible logic. Section 3 discusses an existing implementation of defeasible logic over stratified rule sets, to demonstrate the general process of inferring defeasible conclusions in a distributed setting and the problems that arise. The new logic is introduced in Section 4, while Section 5 establishes its theoretical properties. Section 6 describes the implementation and Section 7 the experimental evaluation. We conclude in Section 8 . Proofs are available in the appendices.

\section{Defeasible Logics}

A defeasible theory $D$ is a triple $(F, R,>)$ where $F$ is a finite set of facts (literals), $R$ a finite set of rules, and $>$ a superiority (or priority) relation (a binary acyclic relation) on $R$, specifying 
when one rule overrides another, given that both are applicable. Rules and facts may be labelled, to enable reference to them. The set of labels is denoted by $\Lambda(D)$.

A rule $r$ consists (a) of its antecedent (or body) $A(r)$ which is a finite set of literals, (b) an arrow, and, (c) its consequent (or head) $C(r)$ which is a literal. There are three types of rules: strict rules, defeasible rules and defeaters represented by a respective arrow $\rightarrow, \Rightarrow$ and $\leadsto$. Strict rules are rules in the classical sense: whenever the premises are indisputable (e.g., facts) then so is the conclusion. Defeasible rules are rules that can be defeated by contrary evidence. Defeaters are rules that cannot be used to draw any conclusions; their only use is to prevent some conclusions.

A literal is a possibly negated predicate symbol applied to a sequence of variables and constants. We will require that any variable in the head of a rule also occurs in the body, and that every fact is variable-free, a property known as range-restricted ${ }^{1}$ Given a fixed finite set of constants, any rule is equivalent to a finite set of variable-free rules, and any defeasible theory is equivalent to a variable-free defeasible theory, for the purpose of semantical analysis. We refer to variablefree defeasible theories, etc as propositional, since there is only a syntactic difference between such theories and true propositional defeasible theories. Consequently, we will formulate definitions and semantical analysis in propositional terms. However, for computational analyses and implementation we will also address defeasible theories that are not propositional.

Given a set $R$ of rules, we denote the set of all strict rules in $R$ by $R_{s}$, and the set of strict and defeasible rules in $R$ by $R_{s d} . R[q]$ denotes the set of rules in $R$ with consequent $q$. If $q$ is a literal, $\sim q$ denotes the complementary literal (if $q$ is a positive literal $p$ then $\sim q$ is $\neg p$; and if $q$ is $\neg p$, then $\sim q$ is $p$ ). A conclusion takes the forms $+d q$ or $-d q$, where $q$ is a literal and $d$ is a tag indicating which inference rules were used. Given a defeasible theory $D,+d q$ expresses that $q$ can be proved via inference rule $d$ from $D$, while $-d q$ expresses that it can be established that $q$ cannot be proved from $D$.

\section{Example 1}

To demonstrate defeasible theories, we consider the representation of the Tweety problem as a defeasible theory. The defeasible theory $D$ consists of the rules and facts

$$
\begin{array}{rrrc}
r_{1}: & \operatorname{bird}(X) & \Rightarrow & f l y(X) \\
r_{2}: & \operatorname{penguin}(X) & \Rightarrow & \neg f l y(X) \\
r_{3}: & \operatorname{penguin}(X) & \rightarrow & \operatorname{bird}(X) \\
e: & \operatorname{bird}(\text { eddie }) & & \\
f: & \text { penguin(tweety) } & &
\end{array}
$$

and a priority relation $r_{2}>r_{1}$.

Here $r_{1}, r_{2}, r_{3}, e, f$ are labels and $r_{3}$ is (a reference to) a strict rule, while $r_{1}$ and $r_{2}$ are defeasible rules, and $e$ and $f$ are facts. Thus $F=\{e, f\}, R_{s}=\left\{r_{3}\right\}$ and $R_{s d}=R=\left\{r_{1}, r_{2}, r_{3}\right\}$ and $>$ consists of the single tuple $\left(r_{2}, r_{1}\right)$. The rules express that birds usually fly $\left(r_{1}\right)$, penguins usually dont fly $\left(r_{2}\right)$, and that all penguins are birds $\left(r_{3}\right)$. In addition, the priority of $r_{2}$ over $r_{1}$ expresses that when something is both a bird and a penguin (that is, when both rules can fire) it usually cannot fly (that is, only $r_{2}$ may fire, it overrules $r_{1}$ ). Finally, we are given the facts that eddie is a bird and tweety is a penguin.

1 This is not a requirement of defeasible theories, it simply eases discussion and implementation. It is a common requirement in work on deductive elements of databases, and is not very restrictive in practice. 
As an example of a defeasible logic, in (Antoniou et al. 2001) a defeasible logic now called $D L(\partial)$ is defined with the following inference rules, phrased as conditions on proof ${ }^{2}$

$+\Delta)$ If $P(i+1)=+\Delta q$ then either

(1) $q \in F$; or

(2) $\exists r \in R_{s}[q] \forall a \in A(r),+\Delta a \in P[1 . . i]$.
$-\Delta)$ If $P(i+1)=-\Delta q$ then

(1) $q \notin F$, and

(2) $\forall r \in R_{s}[q] \exists a \in A(r),-\Delta a \in P[1 . . i]$.

These two inference rules concern reasoning about definitive information, involving only strict rules and facts. They define conventional monotonic inference. The next rules refer to defeasible reasoning.

$+\partial)$ If $P(i+1)=+\partial q$ then either

(1) $+\Delta q \in P[1 . . i]$; or

(2) The following three conditions all hold.

$$
\begin{aligned}
& \text { (2.1) } \exists r \in R_{s d}[q] \forall a \in A(r), \\
& \quad+\partial a \in P[1 . . i] \text {, and } \\
& \text { (2.2) }-\Delta \sim q \in P[1 . . i] \text {, and } \\
& \text { (2.3) } \forall s \in R[\sim q] \text { either } \\
& \text { (2.3.1) } \exists a \in A(s),-\partial a \in P[1 . . i] ;
\end{aligned}
$$

or

(2.3.2) $\exists t \in R_{s d}[q]$ such that

$\forall a \in A(t),+\partial a \in P[1 . . i]$, and

$$
t>s \text {. }
$$

- $\partial)$ If $P(i+1)=-\partial q$ then

(1) $-\Delta q \in P[1 . . i]$, and

(2) either

(2.1) $\forall r \in R_{s d}[q] \exists a \in A(r)$,

$-\partial a \in P[1 . . i]$; or

(2.2) $+\Delta \sim q \in P[1 . . i]$; or

(2.3) $\exists s \in R[\sim q]$ such that

(2.3.1) $\forall a \in A(s),+\partial a \in P[1 . . i]$,

and

(2.3.2) $\forall t \in R_{s d}[q]$ either

$\exists a \in A(t),-\partial a \in P[1 . . i]$; or

$\operatorname{not}(t>s)$.

$+\partial q$ is a consequence of a defeasible theory $D$ if there is a proof containing $+\partial q$.

In the $+\partial$ inference rule, (1) ensures that any monotonic consequence is also a defeasible consequence. (2) allows the application of a rule (2.1) with head $q$, provided that monotonic inference provably cannot prove $\sim q(2.2)$ and every competing rule either provably fails to apply (2.3.1) or is overridden by an applicable rule for $q$ (2.3.2). The $-\partial$ inference rule is the strong negation (Antoniou et al. 2000) of the $+\partial$ inference rule. It establishes when a literal is provably not provable in the logic.

To demonstrate these inference rules, we apply them to the Tweety defeasible theory in the previous example.

\section{Example 2}

We infer $+\Delta$ penguin(tweety) by application of (1) of the $+\Delta$ inference rule, and then $+\Delta$ bird (tweety) by application of (2) of that rule. We also infer $+\Delta$ bird (eddie). From the $-\Delta$ inference rule we infer $-\Delta$ penguin (eddie $),-\Delta f l y($ eddie $),-\Delta \neg f l y($ eddie $),-\Delta f l y($ tweety $)$, and $-\Delta \neg f l y$ (tweety), establishing that these literals cannot be definitely established.

All $+\Delta$ conclusions can also be derived defeasibly, using (1) of the $+\partial$ inference rule. Also note that - Dpenguin(eddie) is derived because $-\Delta$ penguin(eddie) and there is no (instance of) a rule with head penguin (eddie), so (1) and (2.1) of the $-\partial$ inference rule are satisfied. Consequently, we can infer $-\partial \neg f l y($ eddie) because (1) and (2.1) of the $-\partial$ inference rule are satisfied by $-\Delta \neg f l y(e d d i e)$ and $-\partial p e n g u i n(e d d i e)$. Finally, we can now infer $+\partial f l y($ ddie $)$ by (2) of the $+\partial$ inference rule because $r_{1}$ and $+\partial b i r d(e d d i e)$ combine to satisfy (2.1),

2 Here, $D$ is a defeasible theory $(F, R,>), q$ is a variable-free literal, $P$ denotes a proof (a sequence of conclusions constructed by the inference rules), $P[1 . . i]$ denotes the first $i$ elements of $P$, and $P(i)$ denotes the $i^{t h}$ element of $P$. 
$-\Delta \neg f l y($ eddie) satisfies (2.2), and (2.3) is satisfied because the only (instance of a) rule for fly(eddie), $r_{2}$, has penguin(eddie) in its body, and we derived - Dpenguin(eddie).

In contrast, we infer $+\partial \neg f l y\left(\right.$ tweety) because $r_{2}$ and $+\partial$ penguin(tweety) satisfy (2.1), $-\Delta f l y\left(\right.$ tweety) satisfies (2.2), and (2.3) is satisfied because the only rule $s=r_{1}$ with head $\neg f l y$ (tweety) is overruled by $t=r_{2}$ in (2.3.2) using + dpenguin(tweety). Without the priority statement, we would not infer $+\partial \neg f l y$ (tweety), and instead infer $-\partial \neg f l y$ (tweety) (as well as $-\partial f l y($ tweety)), thus being unable to come to any positive conclusion about the ability of tweety to fly.

A tag/inference rule $d$ in a logic is consistent if, for every defeasible theory $D$ in the logic and every proposition $q$, we do not have both consequences $+d q$ and $+d \neg q$ unless we also have consequences $+\Delta q$ and $+\Delta \neg q$. This property expresses that defeasible reasoning does not cause inconsistencies: any inconsistency in consequences is caused by inconsistency in the monotonic part of the defeasible theory. We say a logic is consistent if its main inference rule is consistent. $D L(\partial)$ is consistent, as are the other logics in (Billington et al. 2010).

\section{Parallel Stratified Defeasible Reasoning}

In order to facilitate the discussion in the following sections, we first need to discuss fundamental notions of parallel stratified defeasible reasoning as presented in (Tachmazidis et al. 2012).

A rule set is stratified if all of its predicates can be assigned a rank such that: (a) no predicate depends on one of equal or greater rank, and (b) no predicate is assigned a rank not equal to its complement. Note that a predicate that is found in the head of a rule depends on the predicates that are found in the body of the same rule.

Consider the following stratified rule set:

$$
\begin{array}{cccr}
r_{1}: & r(X, Z), s(Z, Y) & \Rightarrow & q(X, Y) \\
r_{2}: & t(X, Z), u(Z, Y) & \Rightarrow & \neg q(X, Y) \\
r_{1}>r_{2} & &
\end{array}
$$

where predicates $r(X, Z), s(Z, Y), t(X, Z)$ and $u(Z, Y)$ are assigned to rank 0 , while both $q(X, Y)$ and $\neg q(X, Y)$ are assigned to rank 1 .

Predicates that are assigned to rank 0 do not appear in the head of any rule, and thus, only a transformation of given facts into $+\Delta$ and $+\partial$ conclusions is required. Given the facts $r(a, b)$, $s(b, b), t(a, e), u(e, b)$ and $u(e, g)$, this transformation will create the following conclusions (assuming a key-value storage, where the key stores the conclusion itself while the value stores the knowledge about the conclusion):

$$
\begin{aligned}
\langle r(a, b),(+\Delta,+\partial)\rangle & <s(b, b),(+\Delta,+\partial)> \\
\langle t(a, e),(+\Delta,+\partial)> & <u(e, b),(+\Delta,+\partial)> \\
& <u(e, g),(+\Delta,+\partial)>
\end{aligned}
$$

For rank 1, defeasible reasoning needs to be performed in order to resolve the conflict between $q(X, Y)$ and $\neg q(X, Y)$. Due to the nature of defeasible reasoning, parallel reasoning is performed in two passes. The first pass computes applicable rules for $q(X, Y)$ and $\neg q(X, Y)$. Notice that unlike monotonic reasoning, in defeasible reasoning applicable rules might not lead to new conclusions. Hence, the second pass performs the actual defeasible reasoning and computes for each literal whether it is definitely or defeasibly provable.

The following is based on a distributed system with two nodes. However, the same process 
is applicable to any parallel and distributed setting. Note that in order to perform parallel and distributed defeasible reasoning, each node requires a complete knowledge of the given rule set, thus enabling both parallel rule applications (first pass) and parallel defeasible reasoning (second pass). Consider the following distribution of the aforementioned conclusions (for the sake of readability, all knowledge is assumed to be stored in memory):

$$
\begin{array}{ll}
\text { node } 1 & \text { node } 2 \\
<r(a, b),(+\Delta,+\partial)> & <s(b, b),(+\Delta,+\partial)> \\
<t(a, e),(+\Delta,+\partial)> & <u(e, b),(+\Delta,+\partial)> \\
& <u(e, g),(+\Delta,+\partial)>
\end{array}
$$

Considering the first pass, namely computing applicable rules, joins on common arguments for rule $r_{1}$ (resp. $r_{2}$ ) can only be performed if literals $r(a, b)$ and $s(b, b)$ (resp. $t(a, e), u(e, b)$ and $u(e, g)$ ) are located in the same node, performing joins on argument $b$ (resp. argument $e$ ). Thus, the existing knowledge needs to be shuffled as follows:

$$
\begin{aligned}
& \text { node } 1 \quad \text { node } 2 \\
& \langle r(a, b),(+\Delta,+\partial)>\quad\langle t(a, e),(+\Delta,+\partial)> \\
& <s(b, b),(+\Delta,+\partial)>\quad\langle u(e, b),(+\Delta,+\partial)> \\
& <u(e, g),(+\Delta,+\partial)>
\end{aligned}
$$

Note that key-value shuffling for the first pass can be performed according to the hash value of the join argument (argument $Z$ in rules $r_{1}$ and $r_{2}$ ), namely after applying a hash function, argument $b$ is assigned to node 1 while argument $e$ is assigned to node 2 . In this way, joins can be performed locally in each node and in parallel since each node works independently. Such computation will lead to the following knowledge base:

$$
\begin{array}{ll}
\text { node } 1 & \text { node } 2 \\
<r(a, b),(+\Delta,+\partial)> & <t(a, e),(+\Delta,+\partial)> \\
<s(b, b),(+\Delta,+\partial)> & <u(e, b),(+\Delta,+\partial)> \\
<q(a, b),\left(+\partial, r_{1}\right)> & <u(e, g),(+\Delta,+\partial)> \\
& <q(a, b),\left(\neg,+\partial, r_{2}\right)> \\
& <q(a, g),\left(\neg,+\partial, r_{2}\right)>
\end{array}
$$

where $<q(a, b),\left(+\partial, r_{1}\right)>$ means that $q(a, b)$ is supported by rule $r_{1},\left\langle q(a, b),\left(\neg,+\partial, r_{2}\right)\right\rangle$ means that $\neg q(a, b)$ is supported by rule $r_{2}$, and $<q(a, g),\left(\neg,+\partial, r_{2}\right)>$ means that $\neg q(a, g)$ is supported by rule $r_{2}$.

At this point, neither $q(a, b)$ nor $\neg q(a, b)$ can be concluded since the required knowledge for defeasible reasoning is scattered among different nodes. Thus, all knowledge for $q(a, b)$ and $\neg q(a, b)$ must be located in a single node. Hence, the second pass, namely defeasible reasoning, groups all relevant data for each potential conclusion in a single node, with different nodes performing reasoning (in parallel) over different conclusions. Thus, the knowledge will be shuffled as follows:

node 1

$$
\begin{aligned}
& <r(a, b),(+\Delta,+\partial)> \\
& <s(b, b),(+\Delta,+\partial)> \\
& <q(a, b),\left(+\partial, r_{1}\right)> \\
& <q(a, b),\left(\neg,+\partial, r_{2}\right)>
\end{aligned}
$$

node 2

$$
\begin{aligned}
& <t(a, e),(+\Delta,+\partial)> \\
& <u(e, b),(+\Delta,+\partial)> \\
& <u(e, g),(+\Delta,+\partial)> \\
& <q(a, g),\left(\neg,+\partial, r_{2}\right)>
\end{aligned}
$$


This key-value shuffling for the second pass can be performed according to the hash value of the key, namely after applying a hash function, literal $q(a, b)$ is assigned to node 1 while literal $q(a, g)$ is assigned to node 2 . Note that during the second pass only knowledge for literals $q(a, b), \neg q(a, b)$ and $\neg q(a, g)$ is relevant (while literals $r(a, b), s(b, b), t(a, e), u(e, b)$ and $u(e, g)$ are ignored). Notice that conclusions $q(a, b)$ and $\neg q(a, g)$ are computed in parallel by node 1 and node 2 respectively. Finally, after performing defeasible reasoning, the knowledge about applicable rules is replaced by the final conclusions. Thus, the final knowledge base will contain the following conclusions:

$$
\begin{aligned}
& \text { node } 1 \\
& \text { node } 2 \\
& <r(a, b),(+\Delta,+\partial)>\quad<t(a, e),(+\Delta,+\partial)> \\
& <s(b, b),(+\Delta,+\partial)>\quad<u(e, b),(+\Delta,+\partial)> \\
& <q(a, b),(+\partial)>\quad<u(e, g),(+\Delta,+\partial)> \\
& <\neg q(a, g),(+\partial)>
\end{aligned}
$$

For a more elaborate description of parallel stratified defeasible reasoning, readers are referred to (Tachmazidis 2015).

Note that in (Tachmazidis et al. 2012) only positive conclusions are computed in order to ensure scalability. In theory, provable-failure-to-prove (e.g., $-\Delta$ and $-\partial$ inference rules) could be computed by first calculating applicable rules and then applying conflict resolution. However, such computation does not lead to scalable solutions. Consider the following strict rules:

$$
\begin{array}{rrrr}
r^{*}: & p(X, Z), q(Z, Y) & \rightarrow & p(X, Y) \\
r^{\prime}: & q(X, Z), p(Z, V), t(V, Y) & \rightarrow & q(X, Y)
\end{array}
$$

In order to establish that $p(a, b)$ is not definitely provable $(-\Delta)$ every possible instantiated rule needs to be checked, namely for every value of $Z$ either $p(a, Z)$ or $q(Z, b)$ should be established as $-\Delta$. For the aforementioned rule $\left(r^{*}\right)$, if there are $N$ constants in the given dataset, $N$ instantiated rules need to be checked for each $-\Delta$ conclusion.

In general, for $N$ constants in the given dataset and $k$ variables in a given rule that do not appear in the head of the rule (e.g., the variable $Z$ in the aforementioned rule $r^{*}$ ), every $-\Delta$ conclusion (say $-\Delta p(X, Y)$ ) will require $N^{k}$ instantiated rules to be checked. By checking every possible instantiated rule, a significant overhead is introduced that can become prohibitive even for relatively small datasets (e.g. if $N=10^{5}$ and $k=3$ then each conclusion would require the computation of $10^{15}$ rules).

Once all relevant rules are computed, all available information for each literal (such as $p(a, b)$ ) must be processed by a single node (containing all relevant information for the literal to be proved). However, this leads to memory and load balancing problems. For example, if conclusion $-\Delta p(c, b)$ depends on $10^{5}$ instantiated rules (where $N=10^{5}$ and $k=1$, for rule $r^{*}$ ), while conclusion $-\Delta q(c, b)$ depends on $10^{10}$ instantiated rules (where $N=10^{5}$ and $k=2$, for rule $r^{\prime}$ ), then there is a clear difference in the amount of information that needs to be processed by each node (a node computing a $-\Delta p(c, b)$ conclusion is expected to terminate significantly faster than a node computing a $-\Delta q(c, b)$ conclusion).

This problem motivates the definition of a new logic.

\section{A Scalable Defeasible Logic}

The defeasible logic $D L\left(\partial_{\|}\right)$involves three tags: $\Delta$, which we have already seen; $\lambda$, an auxiliary tag; and $\partial_{\|}$, which is the main notion of defeasible proof in this logic. 
For a defeasible theory $D$, we define $P_{\Delta}$ to be the set of consequences in the largest proof satisfying the proof condition $+\Delta$, and call this the $\Delta$ closure. It contains all $+\Delta$ consequences of $D$.

Once $P_{\Delta}$ is computed, we can apply the $+\lambda$ inference rule. $+\lambda q$ is intended to mean that $q$ is potentially defeasibly provable in $D$. The $+\lambda$ inference rule is as follows.

$+\lambda$ : We may append $P(i+1)=+\lambda q$ if either

(1) $+\Delta q \in P_{\Delta}$ or

(2) (2.1) $\exists r \in R_{s d}[q] \forall \alpha \in A(r):+\lambda \alpha \in P(1 . . i)$ and

(2.2) $+\Delta \sim q \notin P_{\Delta}$

Using this inference rule, and given $P_{\Delta}$, we can compute the $\lambda$ closure $P_{\lambda}$, which contains all $+\lambda$ consequences of $D$.

$+\partial_{\|} q$ is intended to mean that $q$ is defeasibly provable in $D$. Once $P_{\Delta}$ and $P_{\lambda}$ are computed, we can apply the $+\partial_{\|}$inference rule.

$+\partial_{\|}$: We may append $P(i+1)=+\partial_{\|} q$ if either

(1) $+\Delta q \in P_{\Delta}$ or

(2) (2.1) $\exists r \in R_{s d}[q] \forall \alpha \in A(r):+\partial_{\|} \alpha \in P(1 . . i)$ and

(2.2) $+\Delta \sim q \notin P_{\Delta}$ and

(2.3) $\forall s \in R[\sim q]$ either

(2.3.1) $\exists \alpha \in A(s):+\lambda \alpha \notin P_{\lambda}$ or

(2.3.2) $\exists t \in R_{s d}[q]$ such that

$\forall \alpha \in A(t):+\partial_{\|} \alpha \in P(1 . . i)$ and $t>s$

The $\partial_{\|}$closure $P_{\partial_{\|}}$contains all $\partial_{\|}$consequences of $D$.

Notice that the structure of the inference rule for $\partial_{\|}$is the same as the structure of the inference rule for $\partial$. However there are important differences to note:

- The inference rule for $\partial_{\|}$uses the closures $P_{\Delta}$ and $P_{\lambda}$ in addition to the single proof $P$ to which it is applied. These closures are pre-computed. In contrast, in $D L(\partial)$ the proof $P$ incorporates both $\Delta$ and $\partial$ conclusions.

- At (2.3.1), the $\partial_{\|}$inference rule refers to $\lambda$ rather than $\partial$; in terms of which conclusions are drawn, this is the most significant variation from $\partial$.

- Furthermore, at (2.2) and (2.3.1), the $\partial_{\|}$inference rule does not use negative tags, such as $-\Delta$, which represent provable failure to prove. Instead, $\partial_{\|}$uses $\notin P$, which represents failure to prove at the meta level, rather than from within the logic. This use of $\notin P$ is only possible because it refers to closures that have already been computed.

Since the proof rules of our logic do not require $-\lambda$ or $-\partial_{\|}$conclusions, we do not present the inference rules for $-\lambda$ and $-\partial_{\|}$here. They are in Appendix C.

It is straightforward to see that $\lambda$ is not consistent. Nevertheless, $D L\left(\partial_{\|}\right)$is consistent. The proof is in Appendix A

\section{Proposition 3}

The inference rule $+\partial_{\|}$is consistent.

Inference rules $\partial$ and $\partial_{\|}$employ the notion of "team defeat", where it doesn't matter which rule overrides an opposing rule, as long as all opposing rules are overridden. This is expressed in (2.3.2). We can also have a version of $\partial_{\|}$with "individual defeat", where all opposing rules must 
be overridden by the same rule, which we denote by $\partial_{\|}^{*}$. The inference rule for $+\partial_{\|}^{*}$ replaces (2.3.2) in $\partial_{\|}$by $r>s$. It, too, is consistent.

To demonstrate the use of $D L\left(\partial_{\|}\right)$, we provide a simple example.

\section{Example 4}

Consider the following defeasible theory describing reachability in a directed graph, where some edges may be broken.

$$
\begin{array}{lrlr}
r: \quad \operatorname{reachable}(X), \operatorname{link}(X, Y) & \rightarrow & \operatorname{reachable}(Y) \\
s: & \operatorname{edge}(X, Y) & \Rightarrow & \operatorname{link}(X, Y) \\
t: & \operatorname{broken}(X, Y) & \Rightarrow & \neg \operatorname{link}(X, Y) \\
& & & \\
\operatorname{reachable}(a) . & & & \\
\operatorname{edge}(a, b) . & \operatorname{edge}(b, c) . & \operatorname{edge}(b, e) . & \operatorname{edge}(c, a) . \\
\text { edge }(c, d) . & \operatorname{edge}(d, e) . & \operatorname{edge}(e, d) . & \operatorname{edge}(f, e) . \\
\text { broken }(c, d) . & \text { broken }(b, e) . & &
\end{array}
$$

with $t>s$.

In this defeasible theory $r$ is a strict rule, $s$ and $t$ are defeasible rules with $t$ overriding $s$ when both are applicable, and there are facts defining the predicates edge and broken, as well as a fact for reachable identifying the starting point for the reachability calculation.

All the facts are known definitely, so they appear in $P_{\Delta}$; there are no other facts in $P_{\Delta}$ because there is no definite information about $l i n k$, and so the only strict rule cannot fire. In addition to all the facts, $P_{\lambda}$ contains $\operatorname{link}(X, Y)$ for each $\operatorname{edge}(X, Y)$ fact, and $\neg \operatorname{link}(X, Y)$ for each broken $(X, Y)$ fact. $P_{\lambda}$ also contains reachable $(X)$ for every $X$ that is reachable from $a$, ignoring the information about broken edges.

$P_{\partial_{||}}$contains all the facts, and $\operatorname{link}(X, Y)$ for each unbroken edge and $\neg \operatorname{link}(X, Y)$ for each broken edge. The superiority relation $t>s$ ensures that $+\partial_{\|} \neg \operatorname{link}(X, Y)$ appears and $+\partial_{\|} \operatorname{link}(X, Y)$ does not appear, for each broken edge. $P_{\partial_{\|}}$also contains reachable $(X)$ for every $X$ that is reachable from $a$, via only unbroken edges.

If we compare $D L\left(\partial_{\|}\right)$with $D L(\partial)$ on this defeasible theory we find that they agree on the defeasible conclusions. Similarly, on the Tweety theory (Examples 1 and 2) the two logics agree on the defeasible conclusions.

The new inference rules provide a scalability advantage when compared to existing inference rules like $\partial$. As pointed out in (Tachmazidis et al. 2012), provable-failure-to-prove (e.g., $-\Delta$ and $-\partial$ inference rules) inhibits the scalability of existing defeasible logics. We saw a little of this in Example 2, where many negative conclusions were needed to derive positive conclusions, but the greater issue arises when rules have variables local to the body of the rule (as discussed in Section 3].

On the other hand, as illustrated in Section 6 the new inference rules that are proposed in this work result in a defeasible logic that is comparable in terms of scalability to existing monotonic logics, and thus able to benefit from available optimizations (readers are referred to (Antoniou et al. 2018) for a comprehensive overview of existing large-scale reasoning methods). 


\section{Properties of the Logic}

In this section we address properties of $D L\left(\partial_{\|}\right)$: the computational complexity of the inference problem, the relative expressiveness of the logic, and the relative inference strength of the logic compared to existing defeasible logics.

\subsection{Computational Complexity}

We first formalize the inference problem for defeasible logics.

The Inference Problem for a Defeasible Logic

\section{Instance}

A defeasible logic $L$, a defeasible theory $T$, a tag/inference rule $d$ and a literal $q$.

\section{Question}

Is $+d q$ derivable from $T$ using the inference rules of $L$ ?

The computational complexity of inference reflects the difficulty of a scalable implementation. We show that $D L\left(\partial_{\|}\right)$has linear complexity for propositional defeasible theories, but exponential for arbitrary defeasible theories. We have three inference rules to consider. As a result of the structure of the inference rules, it is straightforward to compute the consequences of $\Delta$, and then $\lambda$, efficiently.

The inference problem for $\partial$ in propositional defeasible logic has linear complexity (Maher 2001), and we use the same techniques to show that the inference problem for $\partial_{||}$also has linear complexity. The proof is available in Appendix B.

\section{Theorem 5}

The set of all consequences of a propositional defeasible theory can be computed in time linear in the size of the defeasible theory. Consequently, the inference problem for propositional $D L\left(\partial_{\|}\right)$ can be solved in linear time.

However, when variables are permitted in rules the inference problem is EXPTIME-complete.

\section{Corollary 6}

The set of all consequences of a defeasible theory can be computed in time exponential in the size of the defeasible theory. Furthermore, the inference problem for defeasible theories is EXPTIMEcomplete.

From a scalability point of view, the potential for parallelism is important. Unfortunately, the inference problem for $D L\left(\partial_{\|}\right)$is not parallelizible in a theoretical sense, even for propositional defeasible theories. Inference of $\partial_{||}$consequences of propositional defeasible theories is P-complete, which is generally regarded as a sign that the problem is not parallelizible (i.e., not computable in poly-log time with polynomially many processors), unless all polynomial-time problems are parallelizible. Actually, inference of $+\Delta q$ is already P-complete, so all defeasible logics are not parallelizible in this sense. But the proof extends to practically every defeasible logic, even without strict rules.

Theorem 7

The inference problem for propositional defeasible logics is P-complete.

The proof is by reduction of the Horn satisfiability problem, which is P-complete (Cook and Nguyen 2010). 


\subsection{Relative Expressiveness}

Relative expressiveness of defeasible logics is defined in terms of the ability of one logic to simulate another (Maher 2012; Maher 2013), even in the presence of some additions to a theory. The addition of two defeasible theories $\left(F_{1}, R_{1},>_{1}\right)+\left(F_{2}, R_{2},>_{2}\right)$ is $\left(F_{1} \cup F_{2}, R_{1} \cup R_{2},>_{1}\right.$ $\cup>_{2}$ ). Let $\Sigma(D)$ denote the vocabulary of propositions and $\Lambda(D)$ denote the vocabulary of labels for $D$. Given a theory $D$ and a possible simulating theory $D^{\prime}$, an addition $A$ is required to be modular: $\Sigma(A) \cap \Sigma\left(D^{\prime}\right) \subseteq \Sigma(D), \Lambda(D) \cap \Lambda(A)=\emptyset$, and $\Lambda\left(D^{\prime}\right) \cap \Lambda(A)=\emptyset$. This property ensures that the addition $A$ cannot interfere with auxiliary propositions in $D^{\prime}$, nor can it interfere by overruling rules in $D$ or $D^{\prime}$.

A defeasible theory $D_{1}$ in logic $L_{1}$ is simulated by $D_{2}$ in $L_{2}$ with respect to a class $C$ of additions if, for every modular addition $A$ in $C, D_{1}+A$ and $D_{2}+A$ have the same consequences in $\Sigma\left(D_{1}+A\right)$, modulo tag ${ }^{3}$ The classes $C$ of additions considered in (Maher 2013) are: the empty theory, theories consisting only of facts, theories consisting only of rules, and arbitrary theories. These represent progressively stronger notions of simulation.

We say a logic $L_{1}$ can be simulated by a logic $L_{2}$ with respect to a class $C$ if every theory in $L_{1}$ can be simulated by some theory in $L_{2}$ with respect to additions from $C$. We say $L_{2}$ is more (or equal) expressive than $L_{1}$ wrt $C$ if $L_{1}$ can be simulated by $L_{2}$ with respect to $C$. $L_{2}$ is strictly more expressive than $L_{1}$ wrt $C$ if $L_{2}$ is more expressive than $L_{1}$ and $L_{1}$ is not more expressive than $L_{2}$, wrt $C$.

To see the necessity of the restriction to modular additions we present the following example.

\section{Example 8}

Consider a conventional defeasible logic $L_{1}$ that is to be simulated by a similar logic $L_{2}$ that allows only two literals in the body of a rule. A theory $D_{1}$ consisting of a single rule

$$
r: \quad b_{1}, b_{2}, b_{3} \Rightarrow h
$$

in $L_{1}$ might be represented as $D_{2}$ :

$$
\begin{array}{lll}
s: & b_{1}, b_{2} & \Rightarrow \\
r: & t m p, b_{3} & \Rightarrow
\end{array}
$$

in $L_{2}$. However, if an addition $A$ were permitted to include the fact $t m p$ (and $b_{3}$ ) then $D_{1}+A$ cannot infer $h$, but $D_{2}+A$ can infer $h$. Similarly, if $A$ contains facts $b_{1}, b_{2}, b_{3}$ and

$$
s^{\prime}: \quad \Rightarrow \quad \neg t m p
$$

with $s^{\prime}>s$ then $D_{1}+A$ can infer $h$, but $D_{2}+A$ cannot, because $r^{\prime}$ overrules $r$. In either case, $L_{2}$ does not simulate $L_{1}$, despite the close similarity of the two logics.

Thus if non-modular additions were permitted, only simulations that do not use auxiliary predicates and labels are possible, and the notion of relative expressiveness would be useless.

In this section we investigate the relative expressiveness of $D L\left(\partial_{\|}\right)$. We first show that every theory in $D L(\partial)$ can be simulated in $D L\left(\partial_{\|}\right)$. On the other hand, $D L(\partial)$ cannot simulate $D L\left(\partial_{\|}\right)$. In fact, there is a single defeasible theory whose behaviour in $D L\left(\partial_{\|}\right)$cannot be simulated in $D L(\partial)$. Thus $D L(\partial)$ is less expressive than $D L\left(\partial_{\|}\right)$.

3 That is, $D_{1}$ in $L_{1}$ might produce $+d_{1} q$ while $D_{2}$ in $L_{2}$ produces $+d_{2} q$, due to different inference rules in the different logics, but the set of literals $q$ that are derived is the same. 
Theorem 9

$D L(\partial)$ is strictly less expressive than $D L\left(\partial_{\|}\right)$when there are no additions. More specifically,

- every defeasible theory in $D L(\partial)$ can be simulated by a defeasible theory in $D L\left(\partial_{\|}\right)$

- there is a defeasible theory $D$ whose consequences in $D L\left(\partial_{\|}\right)$cannot be expressed by any defeasible theory in $D L(\partial)$

The argument for the second part is based on the following defeasible theory $D$ :

$$
\begin{array}{rr} 
& \Rightarrow \quad p \\
\neg p & \rightarrow \quad \neg p
\end{array}
$$

with empty superiority relation.

$+\lambda p$ and $+\partial_{\|} p$ are consequences of $D$, as is $-\Delta p$, while $-\Delta \neg p$ is not a consequence. However, there is no defeasible theory $D^{\prime}$ in which $+\partial p$ and $-\Delta p$ are consequences but $-\Delta \neg p$ is not. See the proof in Appendix C for details. The argument for the second part applies equally to logics $D L\left(\partial^{*}\right), D L(\delta), D L\left(\delta^{*}\right)$ (defined in (Billington et al. 2010)) because their inference conditions all have the structure that is used in the proof. Essentially, this result arises from the fact that inference in $D L\left(\partial_{\|}\right)$uses, for (2.2), the condition $+\Delta \sim q \notin P_{\Delta}$, whereas the usual defeasible logics use $-\Delta \sim q \in P_{\Delta}$. Thus all these logics are not more expressive than $D L\left(\partial_{\|}\right)$, under any kind of addition.

This theorem suggests that defeasible theories in $D L(\partial)$ (and other logics) could be transformed into theories of $D L\left(\partial_{||}\right)$, and then executed more scalably. However, the proof does not provide such a transformation. Furthermore, the overhead of such a transformation and the expansion in size of the theory could negate the scalability advantages. Nevertheless, this remains an avenue for future research.

Although $D L\left(\partial_{\|}\right)$is able to simulate $D L(\partial)$ when there are no additions, it is unable to achieve a simulation when rules can be added.

\section{Theorem 10}

$D L\left(\partial_{\| \mid}\right)$is not more expressive than $D L(\partial)$ with respect to addition of rules.

The question of whether $D L\left(\partial_{\|}\right)$can simulate $D L(\partial)$ wrt addition of facts remains open.

\subsection{Relative Inference Strength}

We compare the inference strength of the new inference rules to the rules of existing defeasible logics. We write $d_{1} \subseteq d_{2}$ if, for every defeasible theory $T$ and literal $q$, if $+d_{1} q$ is inferred from $T$ then also $+d_{2} q$ is inferred from $T$. This expresses that $d_{2}$ has greater inference strength than $d_{1}$, in the sense that any literal $d_{1}$ can infer can also be inferred by $d_{2}$. We can also view this inclusion as saying that $d_{1}$ is an under-approximation of $d_{2}$, or that $d_{2}$ is an over-approximation of $d_{1}$. We write $d_{1} \subset d_{2}$ (i.e., the inclusion is strict) if $d_{1} \subseteq d_{2}$ and there is a defeasible theory $T$ and literal $q$ such that $+d_{2} q$ is inferred from $T$ but $+d_{1} q$ is not.

The relationship between the inference rules introduced in this paper and those of other defeasible logics is presented in Figure 1 (We follow the notation of (Governatori and Maher 2017) for the inference rules $\sigma_{X}$.) The figure omits $\partial^{*} \subset \lambda$, which is difficult to include in such a two-dimensional representation. Examples show that all the containments are strict, and no containments are missing. The proof relies on results available in Appendix A. 


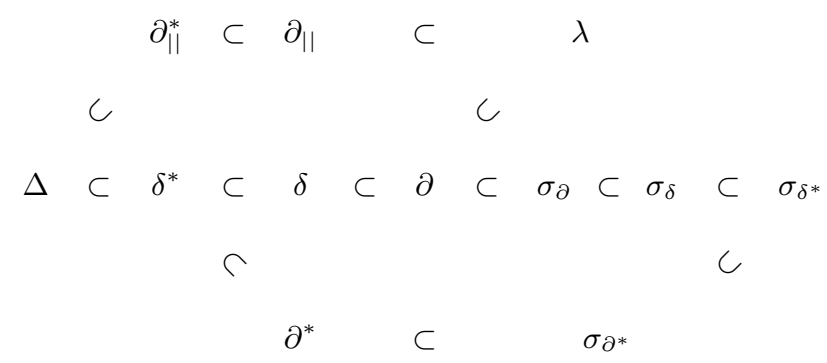

Fig. 1. Ordering of defeasible logic inference rules by relative inference strength. The only unrepresented relation is $\partial^{*} \subset \lambda$.

\section{Theorem 11}

The containments illustrated in Figure 1 hold and are strict. In addition, $\partial^{*} \subset \lambda$ holds. There are no other missing containments in the figure.

In general, relative inference strength provides an indication of how brave/cautious a logic is in making inferences. The results show only that $D L\left(\partial_{\|}\right)$is incomparable to existing logics. Nevertheless, the containment $\partial_{\|}^{*} \subset \partial_{\|}$is noteworthy, since $\partial^{*}$ and $\partial$ are incomparable.

\section{Implementation}

In this section, we present a generic approach for computing the new inference rules by building on previous work. Moreover, we outline the implementation for a real-world case study.

\subsection{Import-Apply-Infer}

An implementation of the new inference rules should first compute the $\Delta$ closure, subsequently the $\lambda$ closure and finally the $\partial_{\|}$closure. It is evident that the $\Delta$ closure computation is conventional rule application, starting from initial facts and repeatedly applying rules until no new conclusion is derived. Large-scale closure computation utilizing parallel and distributed settings over big data posses unique challenges, with a wide range of challenges already addressed in the literature for various logics including Datalog (Condie et al. 2018), $\mathcal{E L}^{+}$(Mutharaju et al. 2015), OWL Horst (Kim and Park 2015) and RDFS (Heino and Pan 2012).

For $\lambda$ and $\partial_{||}$inference rules, we propose a three step method called import-apply-infer, which can be parallelized as depicted in Figure 2. Essentially, the first step (import) reuses existing knowledge that could be considered as facts. Most parallel frameworks provide an efficient data transformation process, thus import's scalability should be considered self evident. The second step (apply) computes all currently applicable rules based on already proved literals. Following data partitioning, data is divided in chunks with each chunk assigned to a node (4 nodes in Figure 2), thus finding matching literals within each node (e.g., $p(X, Z)$ and $q(Z, Y)$ match on argument $Z$ for rule $r^{*}$ in Section 3). Notice that apply follows the same rule application pattern as the first pass in Section 3 . The third step (infer) resolves existing conflicts (e.g., "team defeat"), thus proving and adding new literals to the knowledge base. Notice that infer follows the same conflict resolution pattern as the second pass in Section 3

Upon close inspection, $\lambda$ and $\partial_{\|}$inference rules are variations (in terms of algorithmic computation) of inference rules presented in (Tachmazidis et al. 2012). Thus, the scalability findings 


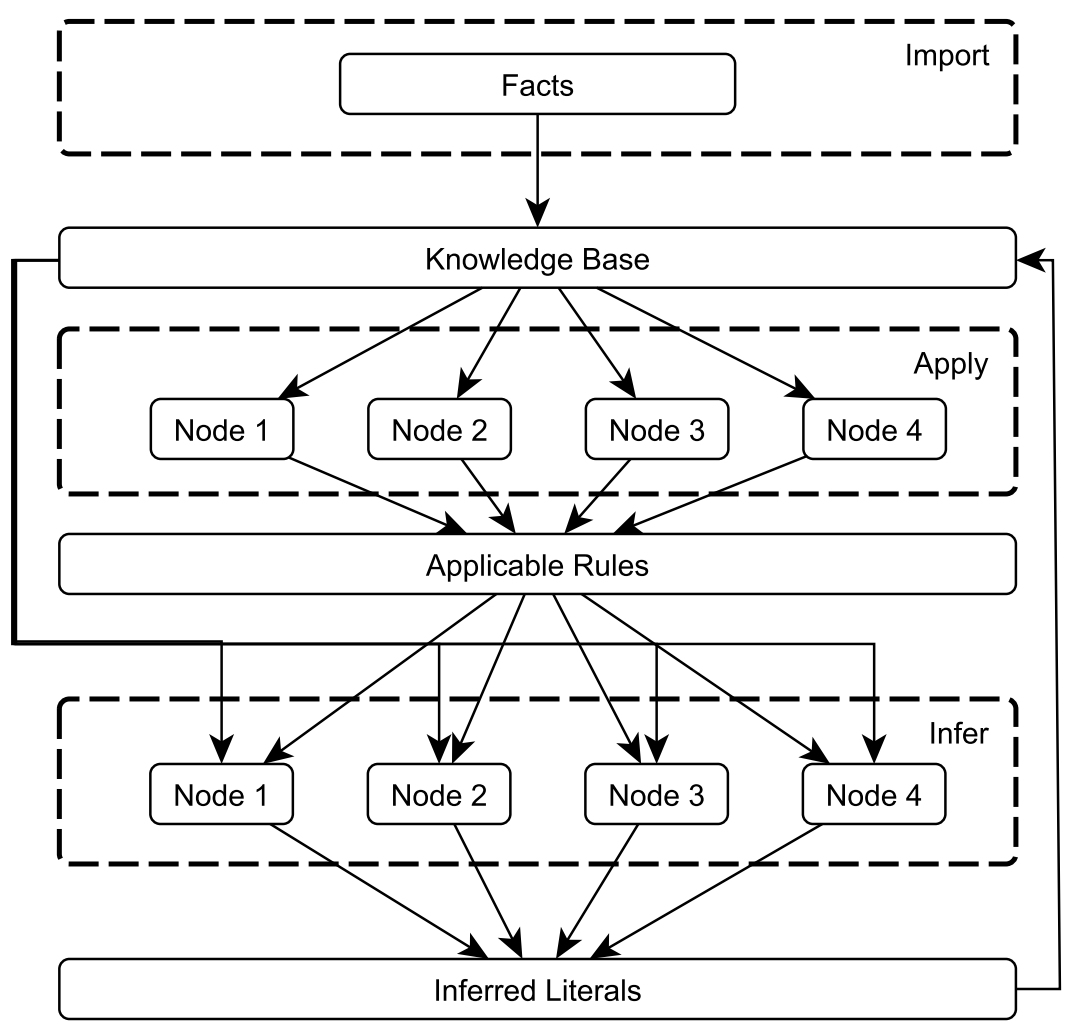

Fig. 2. Parallelizing the Import-Apply-Infer model.

of (Tachmazidis et al. 2012) in terms of a single computation of steps import, apply and infer are applicable to this work as well. Considering closure computation, the import step is computed once at the beginning of the process, while steps apply and infer are computed repeatedly until no new conclusion is derived. Note that a generic implementation of a parallel reasoner is deferred to future work.

For the $\lambda$ closure, clause (1) of the $\lambda$ inference rule corresponds to the import step where literals in $P_{\Delta}$ are treated as given facts, (2.1) of the inference rule corresponds to the apply step as applicable rules are computed based on already proved $\lambda$ predicates, and (2.2) of the inference rule corresponds to the infer step, where a literal $q$ is proved only if $+\Delta \sim q \notin P_{\Delta}$ (with $P_{\Delta}$ already pre-computed). In terms of scalability, the import step requires importing existing knowledge, which is as scalable as the system's data storage, the apply step is as scalable as any rule application (including monotonic reasoning), and the infer step is basic data filtering where knowledge for each literal (both $q$ and $\sim q$ ) is processed in parallel by different nodes in the cluster. Note that for any given rule set the knowledge for a specific literal is significantly smaller than main memory capacity, while the large number of literals ensures a high degree of parallelization and scalability.

For the $\partial_{\|}$closure, clause (1) of the $\partial_{\|}$inference rule corresponds to the import step where literals in $P_{\Delta}$ are treated as given facts, (2.1) of this inference rule corresponds to the apply step as applicable rules are computed based on already proved $\partial_{\|}$predicates, and clauses (2.2) 
and (2.3) correspond to the infer step, where a literal $q$ is proved only if $+\Delta \sim q \notin P_{\Delta}$ (with $P_{\Delta}$ already pre-computed), and either $\exists \alpha \in A(s):+\lambda \alpha \notin P_{\lambda}$ (where $s \in R[\sim q]$ and $P_{\lambda}$ is already pre-computed) or $q$ overrides $\sim q$ through "team defeat". In terms of scalability, $\partial_{\|}$ closure follows a similar pattern as $\lambda$ closure for all three steps. Note that although the infer step for $\partial_{\|}$closure requires more complex computations compared to the $\lambda$ closure, the amount of processed data for each literal is still significantly smaller than main memory capacity, with the large number of literals ensuring a high degree of parallelization and scalability.

\subsection{Apache Spark}

We have used Spark ${ }^{4}$ in our implementations. The main reason is that the platform is very well suited to parallel data processing in distributed environments. It is elastic in terms of both storage (through the use of HDFS) and computation, which is in contrast with the conventional data systems where each node has to be carefully tuned to its specifications (Cheng et al. 2019). This makes Spark be able to greatly simplify the parallel programming of data applications. Namely, developers only need to focus on the design of high-level workflows and can ignore the underlying parallel executions. To handle the complex workflows in our implementation, we have applied Spark SQL (Armbrust et al. 2015) in our data processing. Spark SQL is a module in Apache Spark that integrates relational processing with Spark's functional programming API. Here, we briefly introduce the core abstract of Spark SQL's API - the DataFrame.

A DataFrame in Spark SQL is a distributed collection of rows with the same schema. It can be seen as a table in a relational database while its data is distributed over all computing nodes. A DataFrame can be manipulated and can also perform relational operations over data with existing Spark programs. Currently, DataFrames have supported all the common relational operators, such as projection, filter, join, and aggregations. Moreover, they also enable applications to run SQL queries programmatically and return the result as a DataFrame. Similar to the fundamental data structure of Spark (i.e., RDD), DataFrames are lazy. Namely, in the case that DataFrame object represents a logical plan to compute a dataset, no real parallel execution will occur until an output action such as save is called. This mechanism enables Spark SQL to use data structure information in order to perform rich optimization across all operations that were used to build the DataFrame (Armbrust et al. 2015), which is also the main reason why Spark SQL can provide a highly efficient execution solution for data applications.

\subsection{FDA Use Case with Spark}

The experimental evaluation is based on a FAERS (FDA Adverse Event Reporting System - US Food and Drug Administration) case study, initially developed for RuleRS (Islam and Governatori 2018). More details on this use case are given in Section 7.1. We have implemented the logic using Spark specifically for this use case, and made our code, for the evaluated algorithms in this work, publicly available ${ }^{5}$ The approach reuses fundamental concepts of (Tachmazidis et al. 2012), but is more specific to the ruleset, implements the new logic, and uses Spark. Due to the nature of the FDA rule set, reasoning consists mainly of reporting: (a) obligation conclusions (applicable to all FDA cases) that follow from the given rule set, and (b) identified predicates for

4 https: / / spark.apache.org/

5 https://github.com/longcheng11/dReasoning 


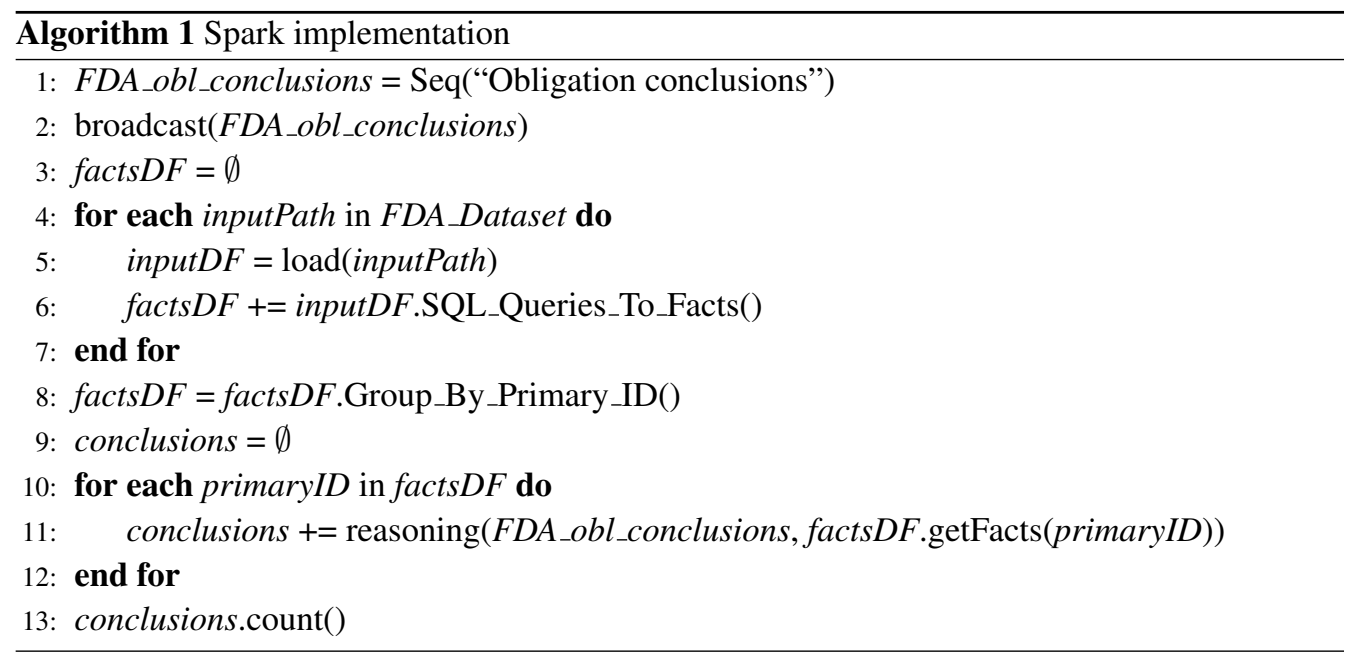

each FDA case. Note that other rule sets might require a more elaborate reasoning implementation.

The basic structure of the implementation is described in Algorithm 1 First, a set of obligation conclusions, that is a set of obligations that need to be concluded for all FDA cases (such as the obligation to report "Patient age", i.e., "obl_report_Patient_age_to_FDA"), is loaded in memory (line 1). Note that obligation conclusions are manually extracted from the FDA rule set. In order to allow each node in the cluster to perform reasoning independently by providing all required information on the given rule set, the set of obligation conclusions needs to be broadcast (line 2). Prior to applying SQL queries, facts are initially set to an empty DataFrame (line 3). Subsequently, each input file in the extracted FDA dataset (lines 4-7) is loaded into a corresponding DataFrame (line 5), and SQL queries are executed (line 6), using Spark SQL, in order to extract predicates (facts) that will be used for reasoning. Spark SQL ensures parallel evaluation of given SQL queries, while the developer needs only to define the queries using the Spark SQL API. Prior to performing reasoning, generated facts are grouped based on their primaryid, namely each FDA case is handled separately (line 8 ). Data grouping is performed in parallel by Spark. Note that lines 3-8 should be considered as the import step.

Considering the reasoning process itself, conclusions are initially set to an empty DataFrame (line 9), while reasoning over each primaryid (in parallel) adds new conclusions (lines 10-12). Essentially, each primaryid is evaluated by a different node in the cluster, thus ensuring parallelism. Note that lines 9-12 should be considered as steps apply and infer. Finally, the number of conclusions is counted (line 13). Counting the number of final conclusions instead of materialising the output allows a better focus on the runtime performance of a given implementation. At the end of Algorithm 1. final conclusions are stored in memory and could be readily used for further processing if required.

\section{Experimental Results}

In this section, we present the results of our experimental evaluation on a commodity cluster. We conduct a quantitative evaluation of our implementation. 
Table 1. Input details

\begin{tabular}{rrrrr}
\hline \hline Copies & Size (GB) & Distinct cases & Rows & Facts \\
\hline \hline 1 & 3 & $5,285,699$ & $43,791,158$ & $96,925,980$ \\
\hline 3 & 9 & $15,857,097$ & $131,373,474$ & $290,777,940$ \\
\hline 6 & 18 & $31,714,194$ & $262,746,948$ & $581,555,880$ \\
\hline 12 & 36 & $63,428,388$ & $525,493,896$ & $1,163,111,760$ \\
\hline \hline
\end{tabular}

Table 2. Number of rows per file

\begin{tabular}{llllll}
\hline \hline DEMO & DRUG & OUTC & REAC & RPSR & Whole \\
\hline \hline $5,285,792$ & $19,087,015$ & $3,649,558$ & $15,525,084$ & 243,709 & $43,791,158$ \\
\hline \hline
\end{tabular}

\subsection{Methodology}

The evaluation of our approach is based on the RuleRS (Islam and Governatori 2018) FAERS (FDA Adverse Event Reporting System - US Food and Drug Administration) case study. The FDA Adverse Event Reporting System (FAERS) is a database that contains adverse event reports, medication error reports and product quality complaints resulting in adverse events that were submitted to FDA. The database is designed to support the FDA's post-marketing safety surveillance program for drug and therapeutic biologic products ${ }^{6}$

Dataset. FAERS publishes quarterly data files 7 , which include:

- DEMO: Demographic and administrative information.

- $D R U G$ : Drug information from the case reports.

- $O U T C$ : Patient outcome information from the reports.

- REAC: Reaction information from the reports.

- RPSR: Information on the source of the reports.

Table 1]describes the details of the used input. The original dataset consists of data published between the third quarter of 2014 and the second quarter of 2018 (a total of four calendar years), which corresponds to 3GB of storage space, 5,285,699 distinct FDA cases (with each case indicated by a unique primaryid), 43,791,158 rows in the consolidated CSV files (for more details see Table 2), and 96,925,980 generated facts by the SQL queries (when all SQL queries were applied). Note that details of applied SQL queries are described below.

The initial dataset allows reasoning over 97M facts, which would not highlight the full potential of the proposed method. For scalability purposes, copies of the aforementioned dataset

\footnotetext{
6 https: / / www. fda.gov/drugs/surveillance/questions-and-answers-fdas-adverseevent-reporting-system-faers

7 https://fis.fda.gov/extensions/FPD-QDE-FAERS/FPD-QDE-FAERS.html
} 
Table 3. Number of SQL queries

\begin{tabular}{lllllll}
\hline \hline DEMO & DRUG & OUTC & REAC & RPSR & Whole \\
\hline \hline & 13 & 5 & 1 & 1 & 1 & 21 \\
\hline \hline
\end{tabular}

Table 4. Number of conclusions (in millions)

\begin{tabular}{rrrrrrl}
\hline \hline Copies & DEMO & DRUG & OUTC & REAC & RPSR & Whole \\
\hline \hline 1 & 310.390 & 291.485 & 150.876 & 280.142 & 10.781 & 335.355 \\
\hline 3 & 931.171 & 874.455 & 452.627 & 840.426 & 32.344 & $1,006.065$ \\
\hline 6 & $1,862.343$ & $1,748.910$ & 905.253 & $1,680.852$ & 64.688 & $2,012.130$ \\
\hline 12 & $3,724.665$ & $3,497.801$ & $1,810.491$ & $3,361.686$ & 129.376 & $4,024.237$ \\
\hline \hline
\end{tabular}

were generated by adjusting the primaryid field, where 3,6 and 12 copies correspond to $291 \mathrm{M}$, $582 \mathrm{M}$ and 1.16 billion facts respectively. Note that the primaryid field is adjusted by appending a counter, namely for 3 copies the following input:

$$
\begin{array}{lll}
\text { primaryid } & \text { caseid } & \text { rpsr_cod } \\
100208273 & 10020827 & \text { FGN }
\end{array}
$$

would be transformed into:

$\begin{array}{lll}\text { primaryid } & \text { caseid } & \text { rpsr_cod } \\ 1002082731 & 10020827 & \text { FGN } \\ 1002082732 & 10020827 & \text { FGN } \\ 1002082733 & 10020827 & \text { FGN }\end{array}$

Note that the first step from 1 copy to 3 copies is counter-intuitive in terms of scalability (not a power of two), however it still provides interpretable results while allowing an evaluation of up to 1.16 billion facts (for 12 copies).

Rule set. The rule set consist of rules that are manually converted from U.S. ELECTRONIC CODE OF FEDERAL REGULATIONS, Title 21: Food and Drugs, PART 310-NEW DRUGS, Subpart D-Records and Reports US Government (2014) As discussed in (Islam and Governatori 2018), the regulations: (a) specify the records and reports concerning adverse drug experiences on marketed prescription drugs for human use without approved new drug applications, and (b) include reporting requirements for Manufacturers, Packers, and Distributors (MPD) and information reported on various life-threatening serious and unexpected adverse drug experience for Individual Case Safety Report (ICSR).

8 https://www.ecfr.gov/cgi-bin/text-idx?SID=7bf64fa0b8f5d9185244a769699c5e13\&mc= true \&node=se21.5.310_1305\&rgn=div8 
Consider for example the provision (as part of informed on ICSRs) prescribing to report electronically to FDA as ICSRs to include "Patient age" while reporting to FDA. Its formal representation in Defeasible Deontic Logic is:

$$
r_{1}:[\mathrm{OAPNP}] \text { report_on_ICSRs_to_FDA }(\mathrm{X}) \Rightarrow[\mathrm{OAPNP}] \text { report_Patient_age_to_FDA(X) }
$$

where [OAPNP] is a deontic operator expressing obligation, while using the defeasible logic as defined in this work we have:

$$
r_{1}: o b l \_r e p o r t \_o n \_I C S R s_{-} t o \_F D A(\mathrm{X}) \Rightarrow \text { obl_report_Patient_age_to_FDA }(\mathrm{X})
$$

Note that obligation conclusions such as obl_report_Patient_age_to_FDA $(X)$ in rule $r_{1}$ are loaded in memory and broadcast to each node in the implementation in order to ensure parallelism (see lines 1-2 in Algorithm 1).

SQL Queries. In (Islam and Governatori 2018) predicate extraction (facts generation) is performed through SQL queries over a PostgreSQL database while in our experiments such SQL queries are part of the implementation using Spark SQL. Each query represents a single predicate, which is eventually passed as an input parameter to the reasoner. Note that the reasoner is implemented using Spark, based on Algorithm 1

The following query illustrates sample test predicates ICSRs_contain_Patient_age in PostgreSQL:

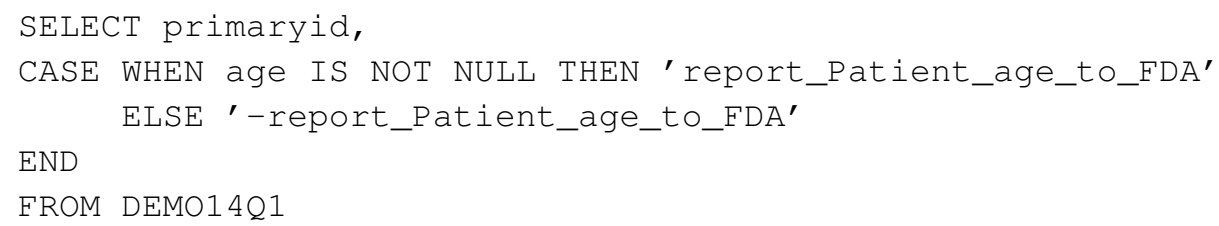

Such query evaluation can be implemented using Spark SQL by loading in memory a DataFrame, called demoDF, containing records found in file DEMO where attribute age must be defined in a given row. The aforementioned SQL query can be translated in Spark SQL as follows (note that in this work only positive literals are relevant):

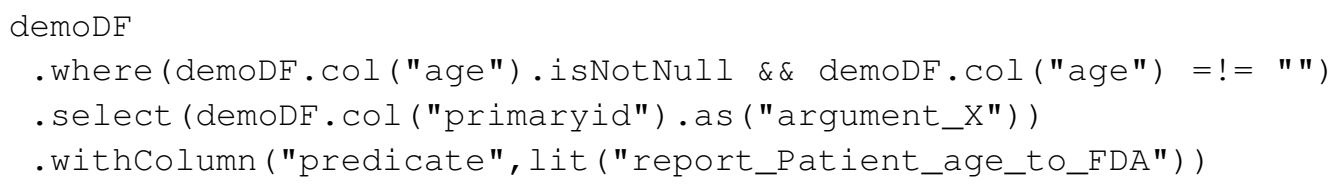

The following input (note that file DEMO contains more columns, which are not included below for readability purposes):

$$
\begin{array}{lllll}
\text { primaryid } & \text { caseid } & \ldots & \text { age } & \ldots \\
100051922 & 10005192 & \ldots & 21 & \ldots
\end{array}
$$

would be transformed into:

$$
\begin{array}{ll}
\operatorname{argument}(X & \text { predicate } \\
100051922 & \text { report_Patient_age_to_FDA }
\end{array}
$$

which essentially represents the fact report_Patient_age_to_FDA(100051922).

The number of executed queries for each file is included in Table 3 , where it is clear that the majority of queries are executed over files DEMO and DRUG. Note that all SQL queries are included in our publicly available implementation (see Section 6.3. 


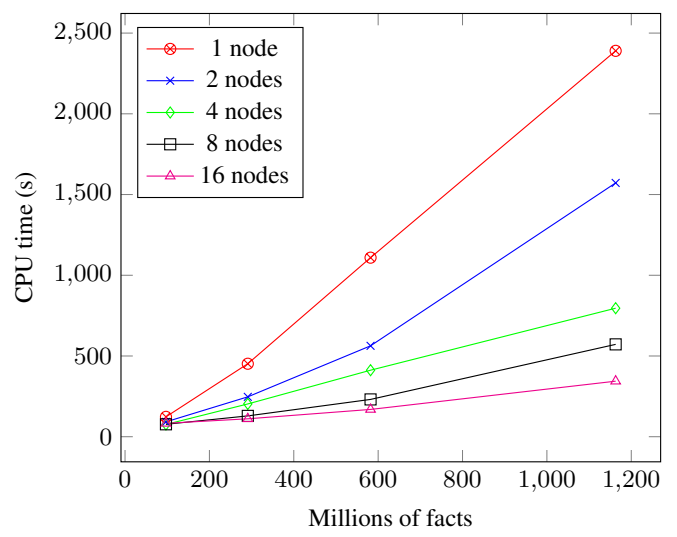

Fig. 3. Time in seconds as a function of number of facts, for various numbers of nodes.
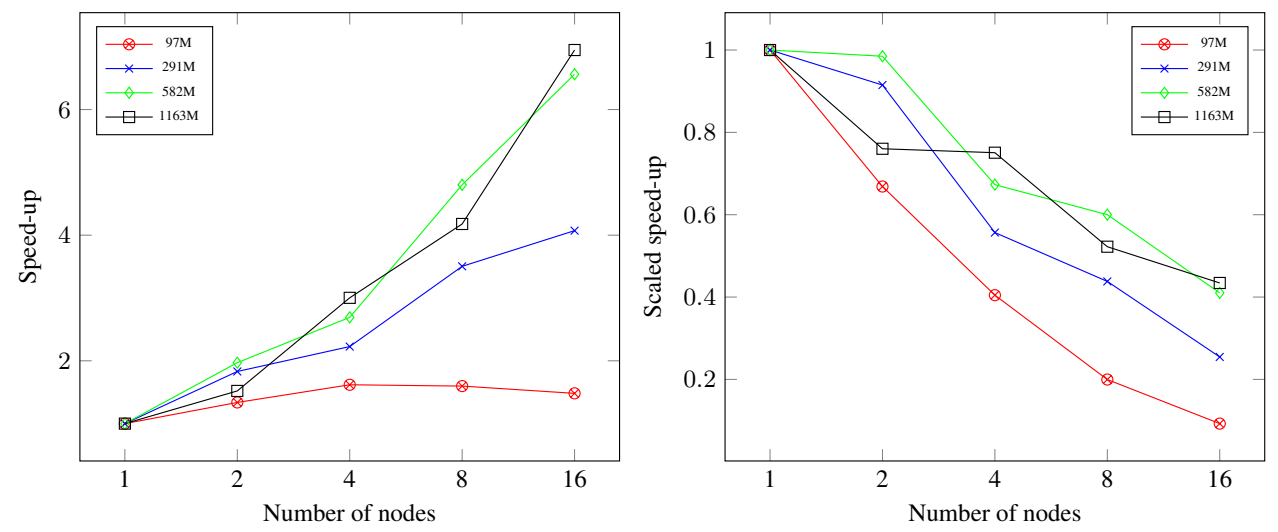

Fig. 4. Speed-up and scaled speed-up as a function of numbers of nodes, for various numbers of facts.

All rules are transformed into the notation defined in this work with the reasoner being implemented in Spark specifically for this rule set. Both fact extraction (Spark SQL) and reasoning (Spark) are implemented within a single job as described in Algorithm 1 (see Section 6.3).

\subsection{Platform}

Our evaluation platform is the Kay supercomputer located at Irish Centre for High-End Computing (ICHEC). We choose up to 17 nodes from the system, and each node we have used contains a 20-core Intel Xeon Gold 6148 (Skylake) processor running at 2.4GHz with 192GB of RAM and a single 400GB SSD local disk. The operating system is Linux kernel version 3.10.0-693 and the software stack consists of Spark version 2.3.1, Hadoop version 2.7.3, Scala version 2.11.8 and Java version 1.8.0_191.

For Spark, we set the following system parameters: spark_worker_memory and spark_executor_memory are set to 160GB and spark_worker_cores is to 20. In all our experiments, the operations of input file reading are on the HDFS system using the SSD on each node. 

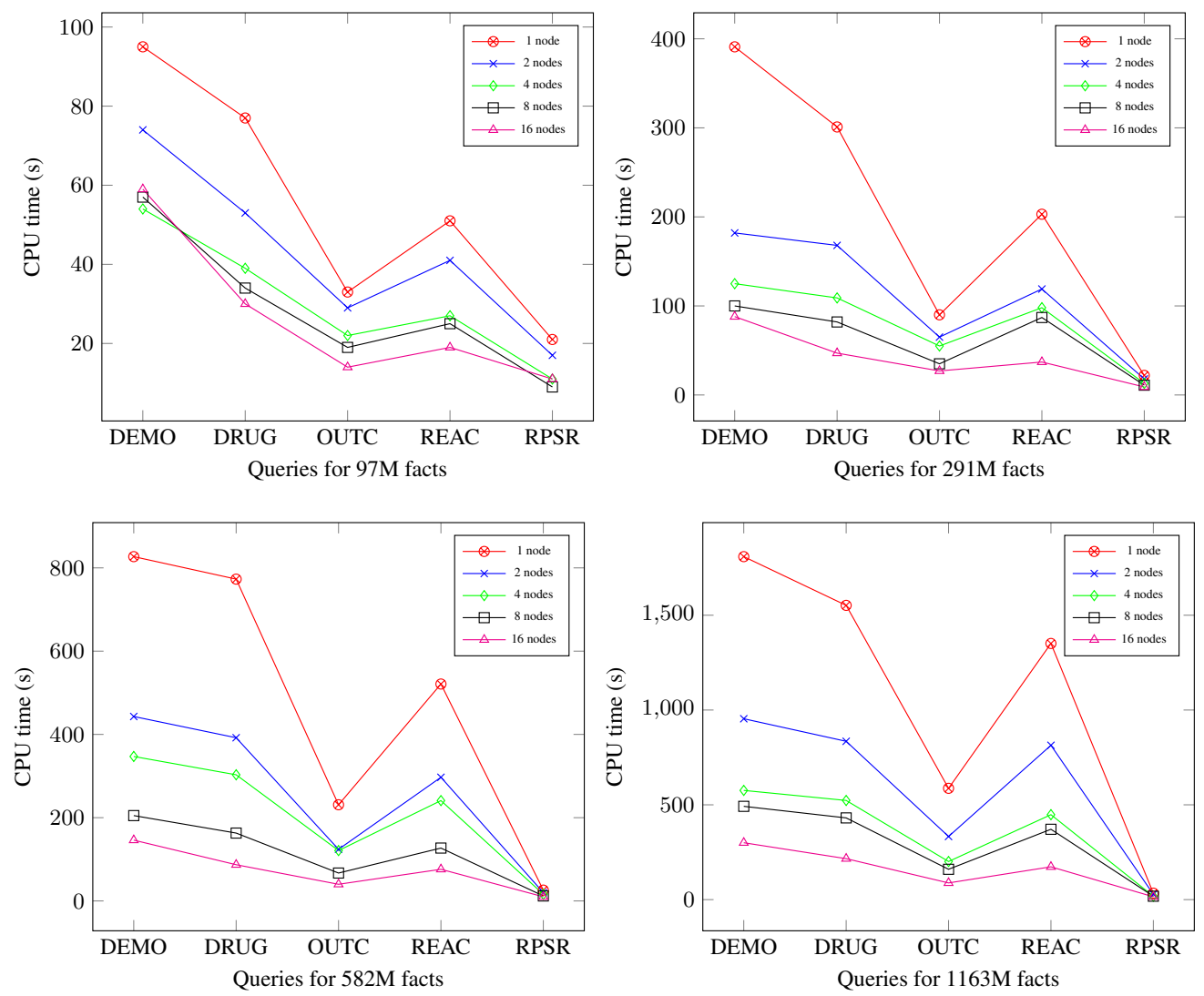

Fig. 5. Time in seconds as a function of queries, for various numbers of facts and nodes.

We measure runtime as the elapsed time from job submission to the job being reported as finished and we record the mean value based on three measurements.

\subsection{Results}

Table 4 provides insight in terms of reasoning. Specifically, it is evident that queries for $D E M O$, $D R U G$ and $R E A C$ are generating comparable numbers of conclusions, OUTC generates approximately half compared to the aforementioned queries, while RPSR generates only a fraction of conclusions. The number of conclusions for each set of queries is a function of the number of rows in the corresponding file (see Table 2) and the number of executed queries (see Table 3 ). However, providing the exact function that would allow an accurate prediction of the number of conclusions, based on the number of rows and executed queries, is out of the scope of this work.

Figure 3 shows the scalability results of the implementation for increasing number of facts. The implementation follows a fairly linear scalability up to 1.16 billion facts when the number of nodes ranges from 1 to 16 . From a practical point of view, the initial dataset (four calendar years) can be processed with 16 nodes in 83 seconds, while 12 copies (corresponding to almost half a century) can be processed in 5 minutes and 44 seconds. For comparison, the initial dataset would require more than 5 days with RuleRS (Islam and Governatori 2018), while auditing the 12 copies 
with RuleRS would require approximately 2 months. Even though the proposed approach in this work cannot be directly compared to RuleRS, given the fact that RuleRS is based on a serial implementation, our results show a significant scalability advantage of the proposed inference rules.

Figure 4 depicts speed-ups and scaled speed-up $9^{9}$ for increasing number of nodes, for various number of facts. It is evident that $97 \mathrm{M}$ of facts is relatively small input in order to show the benefits of parallelization, this is attributed to the fact that the majority of time is dedicated to reading the input. On the other hand, larger inputs highlight the advantages of the distributed implementation. However, the speed-ups are sub-linear regardless of the number of facts or nodes. Nonetheless, the results are encouraging in terms of a proof of concept.

Figure 5 presents the required time in order to execute each set of queries separately (including reasoning over generated facts). The required time in declining order is as follows: DEMO, $D R U G, R E A C, O U T C$ and RPSR. This is consistent with Tables 2 and 4 since larger files require more time to be read, while more conclusions mean both longer reasoning time and more generated facts from the executed queries. Finally, once the input is large enough, there is a clear trend where adding more nodes leads to faster runtimes.

\section{Conclusion and Future Work}

In this paper, we introduced a scalable defeasible logic that allows reasoning over large amounts of data. In particular, we proposed new inference rules for defeasible reasoning, discussed the theoretical properties of the new defeasible logic and ran experiments over an FDA case study (with rules encoding FDA regulations over publicly available FDA datasets). Our experimental results indicate that this method can be applied to billions of facts.

In future work, we plan to develop a generic implementation of a parallel reasoner over the logic we propose in this work. In addition, we plan to study how the proposed inference rules can be extended further in order to model more complex constructs while retaining scalability. In particular, a potential direction could be the introduction of a scalable Defeasible Deontic Logic as an alternative to the one presented in (Governatori et al. 2013). Another direction could be the extension of the proposed defeasible logic in this work to the BOID (Belief, Obligation, Intention, Desire) architecture (Governatori and Rotolo 2008). Such approaches would facilitate reasoning in the legal context, thus providing scalable solutions for processing large amounts of legal documents.

\section{Acknowledgments}

We thank the referees for their comments, which helped improve this paper.

\section{References}

Antoniou, G., Batsakis, S., Mutharaju, R., Pan, J. Z., Qi, G., Tachmazidis, I., Urbani, J., AND Zhou, Z. 2018. A survey of large-scale reasoning on the web of data. Knowledge Eng. Review 33, e21.

\footnotetext{
9 Speed-up is calculated as: $\frac{\text { runtime }_{1 \text { node }}}{\text { runtime }_{\text {Nnodes }}}$, while scaled speed-up is calculated as: $\frac{\text { runtime }_{1 \text { node }}}{N * \text { runtime }_{N n o d e s}}$, where runtime $e_{\text {node }}$ is the required run time for one node, $N$ is the number of nodes and runtime Nnodes $_{\text {is }}$ the required run time for $\mathrm{N}$ nodes.
} 
Antoniou, G., Billington, D., Governatori, G., And Maher, M. J. 1999. On the modelling and analysis of regulations. In Proc. Australasian Conf. on Information Systems. 20-29.

Antoniou, G., Billington, D., Governatori, G., And Maher, M. J. 2000. A flexible framework for defeasible logics. In AAAI/IAAI. AAAI Press / The MIT Press, 405-410.

Antoniou, G., Billington, D., Governatori, G., And Maher, M. J. 2001. Representation results for defeasible logic. ACM Trans. Comput. Log. 2, 2, 255-287.

Armbrust, M., Xin, R. S., Lian, C., Huai, Y., Liu, D., Bradley, J. K., Meng, X., Kaftan, T., Franklin, M. J., Ghodsi, A., ET AL. 2015. Spark sql: Relational data processing in spark. In Proceedings of the 2015 ACM SIGMOD Conference. ACM, 1383-1394.

Billington, D., Antoniou, G., Governatori, G., And Maher, M. J. 2010. An inclusion theorem for defeasible logics. ACM Trans. Comput. Log. 12, 1, 6.

Cheng, L., VAn Dongen, B., And VAn Der Aalst, W. 2019. Scalable discovery of hybrid process models in a cloud computing environment. IEEE Trans. Services Computing.

Condie, T., Das, A., Interlandi, M., Shkapsky, A., Yang, M., And Zaniolo, C. 2018. Scalingup reasoning and advanced analytics on BigData. TPLP 18, 5-6, 806-845.

Cook, S. And NGuYen, P. 2010. Logical Foundations of Proof Complexity. Cambridge University Press.

Dantsin, E., Eiter, T., Gottlob, G., And Voronkov, A. 2001. Complexity and expressive power of logic programming. ACM Comput. Surv. 33, 3, 374-425.

Dowling, W. F. AND GALliER, J. H. 1984. Linear-time algorithms for testing the satisfiability of propositional Horn formulae. J. Log. Program. 1, 3, 267-284.

Gelder, A. V., Ross, K. A., And Schlipf, J. S. 1991. The well-founded semantics for general logic programs. J. ACM 38, 3, 620-650.

Goodman, E. L., Jimenez, E., Mizell, D., Al-Saffar, S., Adolf, B., And Haglin, D. J. 2011. High-Performance Computing Applied to Semantic Databases. In ESWC (2). 31-45.

Governatori, G. ANd Maher, M. J. 2017. Annotated defeasible logic. TPLP 17, 5-6, 819-836.

Governatori, G., Milosevic, Z., And SadiQ, S. W. 2006. Compliance checking between business processes and business contracts. In Tenth IEEE International Enterprise Distributed Object Computing Conference (EDOC 2006). 221-232.

Governatori, G., Olivieri, F., Rotolo, A., And Scannapieco, S. 2013. Computing strong and weak permissions in defeasible logic. J. Philosophical Logic 42, 6, 799-829.

Governatori, G. AND PhAM, D. H. 2009. DR-CONTRACT: an architecture for e-contracts in defeasible logic. IJBPIM 4, 3, 187-199.

Governatori, G. And Rotolo, A. 2008. BIO logical agents: Norms, beliefs, intentions in defeasible logic. Autonomous Agents and Multi-Agent Systems 17, 1, 36-69.

Grosof, B. N., Labrou, Y., AND Chan, H. Y. 1999. A declarative approach to business rules in contracts: courteous logic programs in XML. In Proceedings of the First ACM Conference on Electronic Commerce (EC-99), Denver, CO, USA, November 3-5, 1999. 68-77.

Hashmi, M., Governatori, G., LAm, H., And Wynn, M. T. 2018. Are we done with business process compliance: state of the art and challenges ahead. Knowl. Inf. Syst. 57, 1, 79-133.

HeIno, N. AND PAN, J. Z. 2012. RDFS reasoning on massively parallel hardware. In The Semantic Web - ISWC 2012 - 11th International Semantic Web Conference, Boston, MA, USA, November 11-15, 2012, Proceedings, Part I, P. Cudré-Mauroux, J. Heflin, E. Sirin, T. Tudorache, J. Euzenat, M. Hauswirth, J. X. Parreira, J. Hendler, G. Schreiber, A. Bernstein, and E. Blomqvist, Eds. Lecture Notes in Computer Science, vol. 7649. Springer, 133-148.

ISLAM, M. B. AND Governatori, G. 2018. RuleRS: a rule-based architecture for decision support systems. Artif. Intell. Law 26, 4, 315-344.

KIM, J. AND PARK, Y. 2015. Scalable owl-horst ontology reasoning using SPARK. In 2015 International Conference on Big Data and Smart Computing, BIGCOMP 2015, Jeju, South Korea, February 9-11, 2015. 79-86. 
Leone, N., Allocca, C., Alviano, M., Calimeri, F., Civili, C., Costabile, R., Fiorentino, A., Fuscà, D., Germano, S., Laboccetta, G., Cuteri, B., Manna, M., Perri, S., Reale, K., RicCA, F., Veltri, P., ANd ZANGari, J. 2019. Enhancing DLV for large-scale reasoning. In Logic Programming and Nonmonotonic Reasoning - 15th International Conference, LPNMR 2019, Philadelphia, PA, USA, June 3-7, 2019, Proceedings, M. Balduccini, Y. Lierler, and S. Woltran, Eds. Lecture Notes in Computer Science, vol. 11481. Springer, 312-325.

LiU, C., QI, G., Wang, H., AND Yu, Y. 2011. Large Scale Fuzzy pD* Reasoning Using MapReduce. In 10th International Semantic Web Conference, Bonn, Germany, October 23-27. Lecture Notes in Computer Science, vol. 7031. Springer, 405-420.

Maher, M. J. 2001. Propositional defeasible logic has linear complexity. TPLP 1, 6, 691-711.

MAHER, M. J. 2012. Relative expressiveness of defeasible logics. TPLP 12, 4-5, 793-810.

MAHER, M. J. 2013. Relative expressiveness of defeasible logics II. TPLP 13, 4-5, 579-592.

Maher, M. J., Antoniou, G., And Billington, D. 1998. A study of provability in defeasible logic. In Proc. 11th Australian Joint Conference on Artificial Intelligence. Lecture Notes in Computer Science, vol. 1502. Springer, 215-226.

Martinez-Angeles, C. A., De Castro Dutra, I., Costa, V. S., and Buenabad-Chávez, J. 2013. A datalog engine for GPUs. In Declarative Programming and Knowledge Management - Declarative Programming Days, KDPD 2013, Unifying INAP, WFLP, and WLP, Kiel, Germany, September 11-13, 2013, Revised Selected Papers, M. Hanus and R. Rocha, Eds. Lecture Notes in Computer Science, vol. 8439. Springer, 152-168.

Mutharaju, R., Hitzler, P., Mateti, P., And Lécué, F. 2015. Distributed and scalable OWL EL reasoning. In The Semantic Web. Latest Advances and New Domains - 12th European Semantic Web Conference, ESWC 2015, Portoroz, Slovenia, May 31 - June 4, 2015. Proceedings, F. Gandon, M. Sabou, H. Sack, C. d'Amato, P. Cudré-Mauroux, and A. Zimmermann, Eds. Lecture Notes in Computer Science, vol. 9088. Springer, 88-103.

Oren, E., Kotoulas, S., Anadiotis, G., Siebes, R., ten Teije, A., And van Harmelen, F. 2009. Marvin: Distributed reasoning over large-scale Semantic Web data. J. Web Sem. 7, 4, 305-316.

PraKken, H. 1997. Logical Tools for Modelling Legal Argument: A Study of Defeasible Reasoning in Law. Kluwer Academic Publishers.

Skylogiannis, T., Antoniou, G., Bassiliades, N., Governatori, G., And Bikakis, A. 2007. DR-NEGOTIATE - A system for automated agent negotiation with defeasible logic-based strategies. Data Knowl. Eng. 63, 2, 362-380.

TACHMAZIDIS, I. 2015. Large-scale reasoning with nonmonotonic and imperfect knowledge through mass parallelization. Ph.D. thesis, University of Huddersfield, UK.

TAChmazidis, I. AND Antoniou, G. 2013. Computing the stratified semantics of logic programs over big data through mass parallelization. In Theory, Practice, and Applications of Rules on the Web - 7th International Symposium, RuleML 2013, Seattle, WA, USA, July 11-13, 2013. Proceedings, L. Morgenstern, P. S. Stefaneas, F. Lévy, A. Z. Wyner, and A. Paschke, Eds. Lecture Notes in Computer Science, vol. 8035. Springer, 188-202.

Tachmazidis, I., Antoniou, G., And Faber, W. 2014. Efficient computation of the well-founded semantics over big data. TPLP 14, 4-5, 445-459.

Tachmazidis, I., Antoniou, G., Flouris, G., And Kotoulas, S. 2012. Towards parallel nonmonotonic reasoning with billions of facts. In Principles of Knowledge Representation and Reasoning: Proceedings of the Thirteenth International Conference, KR 2012, Rome, Italy, June 10-14, 2012, G. Brewka, T. Eiter, and S. A. McIlraith, Eds. AAAI Press.

Tachmazidis, I., Antoniou, G., Flouris, G., Kotoulas, S., And McCluskey, L. 2012. Largescale parallel stratified defeasible reasoning. In ECAI 2012 - 20th European Conference on Artificial Intelligence. Including Prestigious Applications of Artificial Intelligence (PAIS-2012) System Demonstrations Track, Montpellier, France, August 27-31 , 2012, L. D. Raedt, C. Bessière, D. Dubois, P. Doherty, P. Frasconi, F. Heintz, and P. J. F. Lucas, Eds. Frontiers in Artificial Intelligence and Applications, vol. 242. IOS Press, 738-743. 
Urbani, J., Kotoulas, S., MaAssen, J., van Harmelen, F., And Bal, H. E. 2012. Webpie: A web-scale parallel inference engine using mapreduce. J. Web Semant. 10, 59-75.

Zhou, Z., Qi, G., LiU, C., Hitzler, P., AND Mutharaju, R. 2013. Scale reasoning with fuzzy-EL+ ontologies based on MapReduce. In Proceedings of the IJCAI-2013 Workshop on Weighted Logics for Artificial Intelligence, WL4AI-2013, Beijing, China, August 2013. 87-93. 


\section{Appendix A Relative Inference Strength}

Proposition 12

$\Delta \subset \partial_{\|}^{*} \subset \partial_{\|} \subset \lambda$

Proof

The first containment follows immediately from (1) of the $\partial_{\|}^{*}$ inference rule. The only difference between $\partial_{\|}^{*}$ and $\partial_{\|}$is in (2.3.2), and the clause for $\partial_{\|}^{*}$ implies the clause for $\partial_{\|}$. The second containment follows. The $\lambda$ inference rule is essentially the $\partial_{||}$inference rule with condition (2.3) omitted. The third containment then follows.

Strictness is shown with straightforward examples. Strictness of the first containment is shown by $D$ consisting only of $\Rightarrow p$. Strictness of the second containment is shown by the standard example distinguishing team and individual defeat: $D$ consists of:

$$
\begin{array}{rlr}
r_{1}: & \Rightarrow & p \\
r_{2}: & \Rightarrow & p \\
r_{3}: & \Rightarrow & \neg p \\
r_{4}: & \Rightarrow & \neg p
\end{array}
$$

with $r_{1}>r_{3}$ and $r_{2}>r_{4}$. Then we can conclude $+\partial_{\|} p$ but not $+\partial_{\|}^{*} p$.

Example 13 shows the strictness of the third containment since $+\lambda q$ is proved but $+\partial_{\|} q$ cannot be proved.

It is straightforward to see that $\lambda$ is not consistent.

\section{Example 13}

Consider the defeasible theory

$$
\begin{array}{rlr}
r: & \Rightarrow & q \\
s: & \Rightarrow & \neg q
\end{array}
$$

with empty superiority relation.

Then we can infer $+\lambda q$ and $+\lambda \neg q$, but cannot infer $+\Delta q$ nor $+\Delta \neg q$. Thus $\lambda$ is not consistent. Furthermore, we cannot infer $+\partial_{\|} q$ nor $+\partial_{\|} \neg q$.

Proposition 14

The inference rule $+\partial_{\| \mid}$is consistent.

Proof

Suppose, for some defeasible theory $D$, and some proposition $q$, that $+\partial_{\|} q$ and $+\partial_{\| \mid} \neg q$ are consequences of $D$.

If $+\Delta \neg q \in P_{\Delta}$ but $+\Delta q \notin P_{\Delta}$ then, when attempting to prove $+\Delta q$, neither (1) nor (2.2) of the $\partial_{\|}$inference rule hold and, thus, $+\Delta q$ cannot be proved. This contradicts our original supposition, so this case cannot occur. Similarly, the case where $+\Delta q \in P_{\Delta}$ but $+\Delta \neg q \notin P_{\Delta}$ cannot occur.

In the third case, neither $+\Delta q$ nor $+\Delta \neg q$ are consequences. Since $+\partial_{\|} q$ is a consequence and (1) does not hold, (2.1) of the $\partial_{\|}$inference rule must hold for some rule $r$ for $q$. Symmetrically, there is a rule $s$ for $\neg q$ such that (2.1) holds. Consequently, by Proposition 12, for each $\alpha \in A(s)$, $+\lambda \alpha \in P_{\lambda}$. Hence, to infer $+\partial_{\|} q$, there must be a rule $t$ for $q$ with $t>s$ and for each $\beta \in A(t)$, $+\partial_{\| \mid} \beta$ is provable and thus $+\lambda \beta \in P_{\lambda}$. But then, to infer $+\partial_{\|} \neg q$, there must be a rule $t^{\prime}$ with 
$t^{\prime}>t$ and for each $\gamma \in A(t),+\partial_{\|} \gamma$ is provable. And so on. This creates an infinite chain of rules, each superior to the previous rule. No rule can be repeated, since $>$ is acyclic. However, the chain cannot be infinite, since the set of rules is finite. This contradiction shows that this case cannot occur.

Thus, by exclusion, both $+\Delta q$ and $+\Delta \neg q$ are consequences, and the result is proved.

It follows immediately from Propositions 3 and 12 that $D L\left(\partial_{\|}^{*}\right)$ also is consistent.

\section{Corollary 15}

The inference rule $\partial_{\|}^{*}$ is consistent.

The next two examples show that $\partial$ and $\partial_{\|}$are incomparable in inference strength.

\section{Example 16}

Consider the defeasible theory

$$
\begin{array}{rrrr}
r: & & \Rightarrow & q \\
s: & \neg q & \rightarrow & \neg q
\end{array}
$$

with $r>s$.

Then $+\Delta \neg q$ cannot be inferred, and so $+\partial_{\|} q$ is inferred. On the other hand, $-\Delta \neg q$ also cannot be inferred, and so $+\partial q$ cannot be inferred. Consequently, $\partial_{\|} \nsubseteq \partial$.

This comes about because of the different treatments of opposing strict inferences in the two inference rules.

\section{Example 17}

Consider the defeasible theory

$$
\begin{aligned}
& r: \quad \Rightarrow \quad q \\
& s: \quad \Rightarrow \quad \neg q \\
& t: \quad \Rightarrow \quad p \\
& u: q \Rightarrow \neg p
\end{aligned}
$$

with no superiority relation.

Then we can infer $-\partial q$ and $+\lambda q$. Consequently, we can infer $+\partial p$, but not $+\partial_{\|} p$. Hence, $\partial \nsubseteq \partial_{\|}$.

This comes about because the inference rules for $+\partial_{\|}$and $+\partial$ differ at (2.3.1): $+\partial_{\|}$requires $+\lambda \alpha \notin P_{\lambda}$ while $+\partial$ requires $-\partial \alpha \in P(1 . . i)$.

Proposition 18

$\partial \subset \lambda$ and $\partial^{*} \subset \lambda$

\section{Proof}

The $\lambda$ inference rule has no condition (2.3), and replaces the condition for $-\Delta \sim q \in P$ for $\partial$ with $+\Delta \sim q \notin P$. By the coherence of defeasible logics (Billington et al. 2010), $+\Delta \sim q \notin P$ is a weaker condition. Hence every inference that $+\partial$ can make can be duplicated by $+\lambda$. The result then follows.

The same argument applies to show that $\partial^{*} \subseteq \lambda$.

Strictness in both cases is straightforward, using the same example and argument (Example 13 . as in the proof of Proposition 12 for the strictness of the third containment.

We establish the lack of any additional containments in Figure 1 using the following three examples. 


\section{Example 19}

Consider the defeasible theory

$$
\begin{array}{rrrr}
r: & & \Rightarrow & q \\
s: & \neg q & \rightarrow & \neg q
\end{array}
$$

with $s>r$.

Then we can infer $+\lambda q$ and $+\partial_{\|}^{*} q$, but not $+\sigma_{\delta^{*}} q$. This arises because the inference rule for $+\lambda$ and $+\partial_{\|}^{*}$ requires only that $+\Delta \sim q$ is not inferred, while the inference rule for $+\sigma_{\delta^{*}}$ must establish $-\delta^{*} \sim q$. In this case, $-\delta^{*} \sim q$ cannot be inferred. Thus $\partial_{\|}^{*} \not \subseteq \sigma_{\delta^{*}}$.

It then follows that $\partial_{\|}^{*} \nsubseteq \mathbb{X}$, for any $X$ considered in (Governatori and Maher 2017) (since $X \subseteq \sigma_{\delta^{*}}($ Billington et al. 2010) $)$, and also $\partial_{\|} \nsubseteq X$ and $\lambda \nsubseteq X$ (since $\left.\partial_{\|}^{*} \subseteq \partial_{\|}\right)$.

We use $\sigma_{X}$ to denote any of the support inference rules $\sigma_{\delta^{*}}, \sigma_{\delta}, \sigma_{\partial^{*}}$, and $\sigma_{\partial}$.

Example 20

Consider the defeasible theory

$$
\begin{array}{rrr}
r: & \Rightarrow & q \\
s: & \rightarrow & \neg q
\end{array}
$$

with no superiority relation.

Then we can infer $+\sigma_{X} q$ but not $+\lambda q$. Thus $\sigma_{X} \nsubseteq \lambda$. This comes about because the inference rules for $+\sigma_{X}$ ignores the possibility of strict inference of $\sim q$, while the inference rule for $+\lambda$ does not.

Hence $\sigma_{X} \nsubseteq \AA \lambda$, for any $X$.

\section{Example 21}

Consider the defeasible theory

$$
\begin{aligned}
& r: \quad \Rightarrow \quad p \\
& s: \quad \Rightarrow \quad \neg p \\
& \Rightarrow \quad q \\
& p \quad \Rightarrow \quad \neg q
\end{aligned}
$$

with $s>r$.

Then we can infer $+\lambda p$, and hence cannot infer $+\partial_{\|} q$. On the other hand, we can infer $-\sigma_{\delta^{*}} p$, since $s>r$, and hence we can infer $+\delta^{*} q$. Thus $\delta^{*} \nsubseteq \partial_{\|}$.

Because $\partial_{\|}^{*} \subseteq \partial_{\|}$and $\delta^{*} \subseteq X$ for every $X$ discussed in (Governatori and Maher 2017) except $\Delta$, we can conclude that neither $\partial_{\|}$nor $\partial_{\|}^{*}$ contains any $X$ discussed in (Governatori and Maher 2017) except $\Delta$.

\section{Theorem 22}

The containments illustrated in Figure 1 hold and are strict. In addition, $\partial^{*} \subset \lambda$ holds. There are no other missing containments in the figure.

\section{Proof}

The containments on the top row of the diagram are established in Proposition 12 The containments on and between the lower two rows are established in (Billington et al. 2010, Governatori and Maher 2017), including their strictness and the lack of any other containments among them. The containments $\partial^{*} \subset \lambda$ and $\partial \subset \lambda$ are established in Proposition 18 .

Example 19 shows that no tag in the lower rows contains a tag in the upper row. Furthermore, 
Example 21 shows that $\partial_{\|}^{*}$ and $\partial_{\|}$do not contain any tag on the lower rows, except for $\Delta$. and Example 20 shows that $\lambda$ does not contain any of the $\sigma_{X}$ tags. Examples showing that containments are strict are straightforward and left to the reader.

\section{Appendix B Complexity}

In this appendix we prove results on the complexity of $D L\left(\partial_{\|}\right)$.

As a result of the structure of the inference rules it is straightforward to compute the consequences of $\Delta$ and $\lambda$ efficiently.

\section{Lemma 23}

The $\Delta$ and $\lambda$ closures, $P_{\Delta}$ and $P_{\lambda}$, of a propositional defeasible theory can be computed in linear time.

Proof

(Sketch) The inference rule for $+\Delta$ is already treated in (Maher 2001). Alternatively, this inference rule is essentially treating strict rules as definite clauses, where negative literals $(\neg p)$ are considered as atoms (e.g. not_p). Such inference can be done in time linear in the size of facts and strict rules (Dowling and Gallier 1984).

Similarly, the inference rule for $+\lambda$ essentially treats strict and defeasible rules as definite clauses, with an extra condition about $\Delta$ consequences. Once the $\Delta$ consequences have been computed, it takes constant extra time for each rule to check the extra condition. Consequently, the inference of $+\lambda$ consequences takes time linear in the size of facts, strict rules and defeasible rules.

Similarly, $-\Delta$ and $-\lambda$ consequences (see Appendix C) are also computed in linear time (although this information is not necessary for the results in this appendix).

The inference problem for propositional $D L(\partial)$ has linear complexity (Maher 2001), and we use the same techniques to show that the inference problem for propositional $D L\left(\partial_{\|}\right)$also has linear complexity.

\section{Theorem 24}

The set of all consequences of a propositional defeasible theory can be computed in time linear in the size of the defeasible theory. Consequently, the inference problem for propositional $D L\left(\partial_{\|}\right)$ can be solved in linear time.

\section{Proof}

(Sketch) We adapt the approach of (Maher 2001). This is possible largely because the structure of the inference rules for $\partial$ and $\partial_{\|}$are the same. First, observe that the transformations of (Antoniou et al. 2001) for $D L(\partial)$ are also correct for $D L\left(\partial_{\|}\right)$. These transformation are used in (Maher $2001)$ to reduce the input defeasible theory $D$ to an equivalent theory in simpler form.

There are three transformations in (Antoniou et al. 2001). The first, regular, separates strict rules from the superiority relation, and it is straightforward to see that this is valid for a wide range of defeasible logics, including $D L\left(\partial_{||}\right)$. The other two, elim_dft and elim_sup, which are used to eliminate defeaters and the superiority relation respectively, employ the same technique to achieve their respective aims: they introduce an intermediate literal in a rule that might be attacked. 
For example, roughly speaking, a rule $B \Rightarrow h$ is replaced by $B \Rightarrow$ temp and temp $\Rightarrow h$, and a defeater $B^{\prime} \leadsto \sim h$ is replaced by $B^{\prime} \Rightarrow \sim$ temp. Similarly, if we have rules $r_{1}: B_{1} \Rightarrow h$ and $r_{2}: B_{1} \Rightarrow \sim h$ with $r_{1}>r_{2}$ then these are replaced by $B_{1} \Rightarrow t e m p_{1}, t e m p_{1} \Rightarrow h$, $B_{2} \Rightarrow t e m p_{2}, t e m p_{2} \Rightarrow \sim h$, and $t e m p_{1} \Rightarrow \neg t e m p_{2}$, where the latter rule encodes $r_{1}>r_{2}$. In each case, when the defeater or overriding rule is active the intermediate literal fails to be proved because it is attacked by another rule, and consequently the application of the original rule is prevented. Because the structure of the inference rules is the same for $D L\left(\partial_{\|}\right)$and $D L(\partial)$, the introduction of intermediate literals and the effect of an attacking rule is the same in both logics. Thus the technique is also correct in $D L\left(\partial_{\|}\right)$.

We have already seen that $P_{\Delta}$ and $P_{\lambda}$ can be computed in linear time. Now we can simplify the transformed version of $D$ and deduce some $\partial_{\|}$consequences.

Let $C$ be a set of consequences, initially $\emptyset$.

1. For each literal $q$ : If $+\Delta q \in P_{\Delta}$ then delete all defeasible rules for $\sim q$, add $+\partial_{\|} q$ to $C$, and delete all occurrences of $q$ from the body of rules.

2. For each literal $q$ : If $+\lambda q \notin P_{\lambda}$ and $q$ occurs in the body of a rule, delete the rule.

3. Delete all strict rules.

Simplification 1 is justified by (2.2) and (1) of the inference rule. Simplification 2 is justified by (2.3.1) of the inference rule, and by Proposition 12 (which implies that such rules cannot be used in (2.1) for $\sim q)$. Simplification 3 is justified because all definite consequences are already available in $P_{\Delta}$ and, as a result of the regular transformation, no other use is made of these rules.

The simplified theory $D^{\prime}$ incorporates the all the effects of references to $P_{\Delta}$ and $P_{\lambda}$. Consequently, the transition system of (Maher 2001) applies also to $D^{\prime}$ with initial consequences $C$, for $D L\left(\partial_{\|}\right)$. In fact, only the transitions numbered $2,4,5$, and 8 are needed, since the remaining transitions involve strict rules or negative tags, though 5 is modified by dropping the reference to $-\Delta \sim q$. The simplifications above can be viewed as variants of transitions: simplification 1 corresponds to transitions 6 and 1; and simplification 2 corresponds to transition 10. Simplification 3 is essentially redundancy removal, given $C$. Furthermore, the data structure used in (Maher 2001) to achieve linear complexity in application of the transition system is also applicable to $D L\left(\partial_{\|}\right)$.

Thus all positive consequences of a propositional defeasible theory $D$ in $D L\left(\partial_{\|}\right)$can be computed in time linear in the size of $D$.

\section{Corollary 25}

The set of all consequences of a defeasible theory can be computed in time exponential in the size of the defeasible theory. Furthermore, the inference problem for defeasible theories is EXPTIMEcomplete.

\section{Proof}

Construct a propositional defeasible theory $D^{\prime}$ from the original defeasible theory $D$ by taking all variable-free instances of all rules using the constants that appear in $D$. Two instances of rules are related by the superiority relation iff the rules of which they are instances are so related. Let $n$ be the maximum number of variables in a rule of $D$ and $c$ be the number of constants in $D$. Then there are at most $c^{n}$ propositional instances of a rule of $D$, and at most $c^{2 n}$ derived superiority statements for each superiority statement in $D$. Since both $n$ and $c$ may be $\mathrm{O}(|D|)$, the size of $D^{\prime}$ is $\mathrm{O}\left(|D|^{2|D|}\right)$, which is $\mathrm{O}\left(2^{p(|D|)}\right)$, for a polynomial $p$. 
$D$ and $D^{\prime}$ have the same consequences. By Theorem 5 the consequences of $D^{\prime}$ can by computed in linear time in the size of $D^{\prime}$, which is EXPTIME in the size of $D$.

The inference problem is shown EXPTIME-complete by reduction of the same problem for Datalog (see (Dantsin et al. 2001), Theorem 4.5). Each Datalog rule is expressed as a defeasible rule. A positive literal is inferred in $D L\left(\partial_{\|}\right)$iff it is inferred in Datalog.

\section{Theorem 26}

The inference problem for propositional defeasible logics is P-complete.

Proof

We show that the inference problem for $+\Delta$ is P-complete, by reduction of the Horn satisfiability problem, which is P-complete (Cook and Nguyen 2010). For completeness, we first specify this problem. A Horn clause is a disjunction of literals containing at most one positive literal.

\section{The Horn Satisfiability Problem}

\section{Instance}

A set $H$ of propositional Horn clauses.

\section{Question}

Is $H$ satisfiable, that is, is there an assignment of Boolean values to propositional variables such that each clause of $H$ evaluates to true?

In the reduction, each of the propositional variables in the Horn satisfiability problem is represented by itself, and we add an extra propositional variable false. For clarity, we write the Horn clauses in the logic programming style.

For every Horn clause of the form

$$
A \leftarrow B_{1}, \ldots, B_{n}
$$

the defeasible theory contains the strict rule

$$
B_{1}, \ldots, B_{n} \quad \rightarrow \quad A
$$

Similarly, for every Horn clause of the form

$$
\leftarrow B_{1}, \ldots, B_{n}
$$

the defeasible theory contains the strict rule

$$
B_{1}, \ldots, B_{n} \rightarrow \text { false }
$$

It is straightforward to show that $+\Delta q$ is inferred by a defeasible logic iff $q$ is true in every model of the definite clause subset of $H$, and $+\Delta$ false is inferred by a defeasible logic iff $H$ is unsatisfiable.

Strict inference is a part of any defeasible logic, so the result applies to all defeasible logics. Even without a separate notion of strict inference, the proof extends easily to any inference rule that allows the chaining of defeasible or strict rules, since the superiority relation and conflicting rules do not arise in the reduction. This includes all defeasible logics we are aware of. 


\section{Appendix C Relative Expressiveness}

In this appendix we prove Theorems 9 and 10

Relative expressiveness involves both positive and negative tags, so we first introduce the inference rules for $-\lambda$ and $-\partial_{\|}$. These inference rules are a kind of negation of the corresponding positive inference, under the Principle of Strong Negation (Antoniou et al. 2000). However, the notion of strong negation must be extended to address expressions of the form $t \alpha \notin P$, which were not considered in (Antoniou et al. 2000). In these cases we define the strong negation of $t \alpha \notin P$ to be $t \alpha \in P$.

The closure $P_{\Delta}$ must be closed under both $+\Delta$ and $-\Delta$ inference rules, that is, it must contain all $+\Delta$ and $-\Delta$ consequences.

The $-\lambda$ inference rule is as follows.

$-\lambda$ : We may append $P(i+1)=-\lambda q$ if both

$$
\begin{aligned}
& \text { (1) }-\Delta q \in P_{\Delta} \text { and } \\
& \text { (2) (2.1) } \forall r \in R_{s d}[q] \exists \alpha \in A(r):-\lambda \alpha \in P(1 . . i) \text { or } \\
& \text { (2.2) }+\Delta \sim q \in P_{\Delta}
\end{aligned}
$$

The $\lambda$ closure $P_{\lambda}$ contains all $+\lambda$ and $-\lambda$ consequences of $D$.

$-\partial_{\|}$: We may append $P(i+1)=-\partial_{\|} q$ if both

(1) $-\Delta q \in P_{\Delta}$ and

(2) (2.1) $\forall r \in R_{s d}[q] \exists \alpha \in A(r):-\partial_{\|} \alpha \in P(1 . . i)$ or

(2.2) $+\Delta \sim q \in P_{\Delta}$ or

(2.3) $\exists s \in R[\sim q]$ such that

$$
\begin{aligned}
& \text { (2.3.1) } \forall \alpha \in A(s):+\lambda \alpha \in P_{\lambda} \text { and } \\
& \text { (2.3.2) } \forall t \in R_{s d}[q] \text { either } \\
& \quad \exists \alpha \in A(t):-\partial_{\|} \alpha \in P(1 . . i) \text { or } t \ngtr s
\end{aligned}
$$

To prove the first part of Theorem 9 we employ an analysis introduced in (Maher et al. 1998, Antoniou et al. 2001). For each proposition $p$ we can identify exactly six different possible outcomes of the proof theory. With each outcome we present a simple theory that achieves this outcome.

$$
\begin{aligned}
\mathrm{A}: & -\Delta p \notin P_{\Delta} \text { and }+\partial_{||} p \notin P_{\partial_{\|}} \\
& p \rightarrow p \\
\mathrm{~B}: & +\partial_{||} p \in P_{\partial_{||}} \text {and }+\partial_{\|} p \notin P_{\Delta} \text { and }-\Delta p \notin P_{\Delta} \\
& \Rightarrow p ; p \rightarrow p \\
\mathrm{C}: & \left.+\Delta p \in P_{\Delta} \text { (and also }+\partial_{||} p \in P_{\partial_{||}}\right) \\
& \rightarrow p \\
\mathrm{D}: & +\partial_{||} p \in P_{\partial_{\|}} \text {and }-\Delta p \in P_{\Delta} \\
& \Rightarrow p \\
\mathrm{E}: & -\Delta p \in P_{\Delta} \text { and }+\partial_{||} p \notin P_{\partial_{||}} \text {and }-\partial_{||} p \notin P_{\partial_{\|}} \\
& p \Rightarrow p \\
\text { F: } & \left.-\partial_{||} p \in P_{\partial_{\mid}} \text {(and also }-\Delta p \in P_{\Delta}\right) \\
& \emptyset, \text { the empty theory }
\end{aligned}
$$

Similarly, there are the same six possibilities for $\neg p$. We can represent the outcomes in terms of a Venn diagram in Figure C1. 


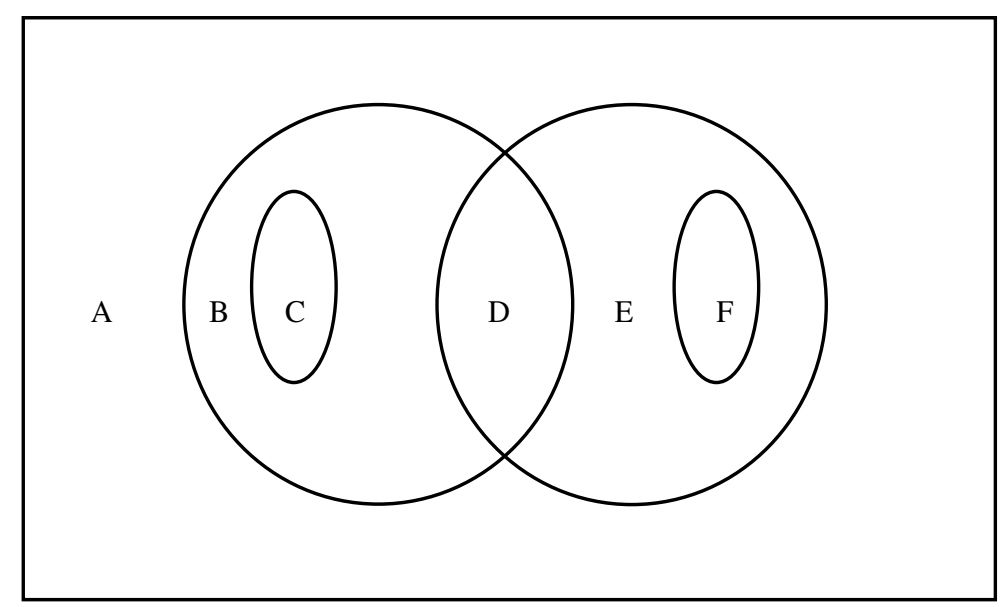

Fig. C 1. Possible outcomes for a single literal in $D L\left(\partial_{\|}\right)$

In Figure C 1, the circle on the left - containing B, C, and D - represents the literals $p$ such that $+\partial_{\|} p$ can be proved, and the ellipse inside it (i.e. C) represents the literals $p$ such that $+\Delta p$ can be proved. The circle on the right - containing $\mathrm{D}, \mathrm{E}$, and $\mathrm{F}$ - represents the literals $p$ such that $-\Delta p$ can be proved, and the ellipse inside it (i.e. F) represents the literals $p$ such that $-\partial_{\|} p$ can be proved.

Due to the relationship between $p$ and $\neg p$, many fewer than the 36 possible combinations are possible outcomes of the proof theory.

In what follows, $p$ ranges over literals and $\sim p$ denotes the complement of $p$. We first establish some simple properties that will eliminate many combinations.

\section{Proposition 27}

Consider a defeasible theory $D$, with $\Delta$ closure $P_{\Delta}$ and $\partial_{||}$closure $P_{\partial_{\| \mid}}$.

1. If $+\Delta p \notin P_{\Delta}$ and $+\Delta \sim p \in P_{\Delta}$ then $+\partial_{\|} p \notin P_{\partial_{\|}}$

2. If $-\Delta p \in P_{\Delta}$ and $+\Delta \sim p \in P_{\Delta}$ then $-\partial_{\|} p \in P_{\partial_{\|}}$

3. If $+\Delta p \notin P_{\Delta}$ and $+\Delta \sim p \notin P_{\Delta}$ then we cannot have both $+\partial_{||} p \in P_{\partial_{||}}$and $+\partial_{\|} \sim p \in$ $P_{\partial \|}$

\section{Proof}

Statements 1 and 2 follow directly from the proof rules for $+\partial_{\|}$and $-\partial_{\|}$. Statement 3 is proved as follows.

Suppose this combination is possible. Then, when applying the inference rule $\partial_{||}$for $p$ (and for $\sim p$ ), (1) does not apply and (2.2) is satisfied. We also must have $+\lambda p$ and $+\lambda \sim p$ because $\partial_{\|} \subseteq \lambda$ and by definition of $\mathrm{D}$ (because we have $+\partial_{\|} p$ and $+\partial_{\|} \sim p$ ). There must be a rule $r$ for $p$ (and one for $\sim p$ ) such that $\forall \alpha \in A(r)+\partial_{\|} \alpha \in P_{\partial_{||}}$. Consequently, there is a rule $s$ for $p$ (and one for $\sim p$ ) such that $\forall \alpha \in A(s)+\lambda \alpha \in P_{\lambda}$. Hence (2.3.1) of inference rule $\partial_{\|}$does not apply, and so (2.3.2) must. That is, there is a rule $r$ for $p$ (and one for $\sim p$ ) such that $\forall \alpha \in A(t)+\partial_{\| \mid} \alpha \in P_{\partial_{\|}}$ and $t>s$. In this way we obtain a chain of rules: for each rule $s_{i}$ for $\sim p$ there is a superior rule $t_{i}$ for $p$, and for each rule $t_{i}$ for $p$ there is a superior rule $s_{i}$ for $\sim p$. Since $D$ is finite and $>$ is acyclic, this can never occur. 


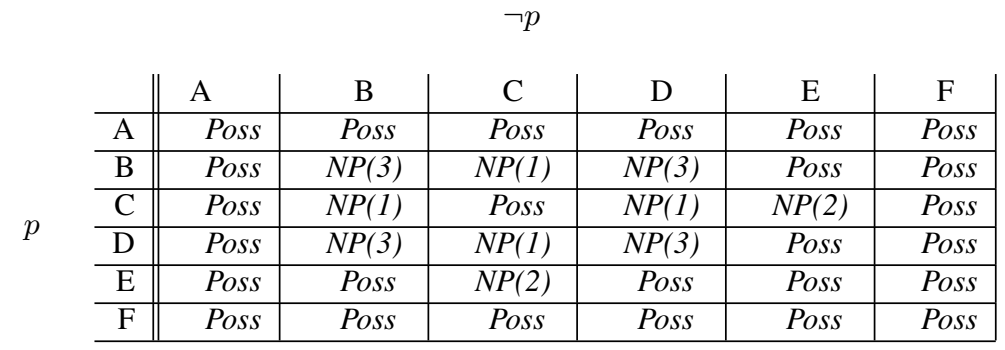

Fig. C 2. Table of all combinations of conclusions for $p$ and $\neg p$ in $D L\left(\partial_{\|}\right)$, indicating whether or not the combination is possible, and, if not, why not.

In terms of the diagram (Figure C1), the properties of the previous proposition have the following effects:

1. If $p$ satisfies $\mathrm{A}, \mathrm{B}, \mathrm{D}, \mathrm{E}$, or $\mathrm{F}$, and $\sim p$ satisfies $\mathrm{C}$ then $p$ satisfies $\mathrm{A}, \mathrm{E}$, or $\mathrm{F}$ (Property 1). Consequently, it is not possible for $p$ to satisfy $\mathrm{B}$ or $\mathrm{D}$, and $\sim p$ to satisfy $\mathrm{C}$.

2. If $p$ satisfies $\mathrm{D}$, E, or $\mathrm{F}$, and $\sim p$ satisfies $\mathrm{C}$ then $p$ satisfies $\mathrm{F}$ (Property 2). Consequently, it is not possible for $p$ to satisfy $\mathrm{D}$ or $\mathrm{E}$, and $\sim p$ to satisfy $\mathrm{C}$.

3. If $p$ satisfies $\mathrm{B}$ or $\mathrm{D}$ and $\sim p$ satisfies $\mathrm{B}$ or $\mathrm{D}$ we have a contradiction. That is, it is not possible for $p$ to satisfy $\mathrm{B}$ or $\mathrm{D}$, and $\sim p$ to satisfy $\mathrm{B}$ or $\mathrm{D}$.

These effects apply for $p$ a positive or negative literal.

In the table in Figure C2 we display the possible combinations of conclusions for a proposition $p$ and its negation $\neg p$. The table is symmetric across the leading diagonal, since the treatment of literals in defeasible logic is independent of the polarity of the literal. Those combinations which are possible are displayed as Poss. Those combinations which are not possible are displayed as $N P(i)$, where $i$ is the property number in Proposition 27 that implies that they are impossible.

For the possible combinations, a sample theory can be exhibited by combining the sample theories for each letter (for $p$ and $\sim p$, respectively). We leave this for the reader to verify.

It is now straightforward to compare this table, for $D L\left(\partial_{\|}\right)$, with the table in (Maher et al. 1998; Antoniou et al. 2001) for $D L(\partial)$. Every combination that is possible for $D L(\partial)$ is also possible for $D L\left(\partial_{\|}\right)$. Thus, for any defeasible theory $D$, and each proposition $p$, we can identify which combination of conclusions $D L(\partial)$ entails and simulate that behaviour with the sample theory for that combination for $p$. This completes the proof of the first part of Theorem 9 Thus we have

Theorem 28

$D L(\partial)$ is less expressive than $D L\left(\partial_{\|}\right)$when there are no additions. More specifically,

- every defeasible theory in $D L(\partial)$ can be simulated by a defeasible theory in $D L\left(\partial_{\|}\right)$

- there is a defeasible theory $D$ whose consequences in $D L\left(\partial_{\|}\right)$cannot be expressed by any defeasible theory in $D L(\partial)$

Proof

The proof of the first part is established by the preceding work in this section. For the second part, let $D$ consist of

$$
\begin{array}{rr} 
& \Rightarrow \\
\neg p & \rightarrow \quad \neg p
\end{array}
$$


with empty superiority relation.

Then $+\lambda p$ and $+\partial_{\|} p$ are consequences of $D$, as is $-\Delta p$, while $-\Delta \neg p$ is not a consequence.

We now show that $D L(\partial)$ cannot simulate this theory. That is, for no defeasible theory $D^{\prime}$ are both $+\partial p$ and $-\Delta p$ consequences, and $-\Delta \neg p$ not a consequence. Suppose $+\partial p$ is a consequence of $D^{\prime}$. Then either (1) $+\Delta p$ or (2) $-\Delta \neg p$ must be consequences of $D^{\prime}$, from the inference condition for $+\partial$. But these contradict the requirements on the $\Delta$-consequences of $D^{\prime}$. Thus $D L(\partial)$ is unable to simulate the consequences of $D$ under $D L\left(\partial_{\|}\right)$.

It is interesting to note that the comparison of tables, identifies several different theories that might be used to establish the second part of Theorem 9. However, they all have a similar structure: a literal $q$ is defeasibly provable, despite a loop for $\sim q$.

Theorem 29

$D L\left(\partial_{\|}\right)$is not more expressive than $D L(\partial)$ with respect to addition of rules.

\section{Proof}

Let $D$ be the empty defeasible theory $(\emptyset, \emptyset, \emptyset)$. The consequences of $D$ in $D L(\partial)$ are $-\partial q$, $-\partial \neg q,-\Delta q$ and $-\Delta \neg q$ for every proposition $q$. Suppose, to achieve a contradiction, that $D^{\prime}$ is a simulation of $D$ wrt addition of rules in $D L\left(\partial_{\|}\right)$. Then $-\Delta q$ and $-\Delta \neg q$ are consequences of $D^{\prime}$. We will consider several additions to $D$.

For the addition $A_{1}$

$$
r_{1}: \quad \Rightarrow \quad q
$$

$D+A_{1}$ has consequences $+\partial q$ and $-\partial \neg q$.

Then $+\partial_{\|} q$ is a consequence of $D^{\prime}+A_{1}$. It is straightforward that $-\Delta q$ and $-\Delta \neg q$ are consequences of $D^{\prime}+A_{1}$. It follows, from the $\partial_{\|}$inference rule, that (2.3.1) or (2.3.2) holds for each rule $s$ for $\neg q$ in $D^{\prime}+A_{1}$.

Note that $+\lambda q \in P_{\lambda}$ for $D^{\prime}+A_{1}$, by Proposition 12. Now, if $+\lambda \alpha \notin P_{\lambda}$ for $D^{\prime}+A_{1}$, for some $\alpha$, then also $+\lambda \alpha \notin P_{\lambda}$ for $D^{\prime}$, because $P_{\lambda}$ monotonically increases with the addition of $+\lambda$ consequences. Furthermore, $r_{1}$ is not superior to any rule in $D^{\prime}$ (by definition of modular addition). Consequently, (2.3.1) or (2.3.2) holds for each rule $s$ for $\neg q$ in $D^{\prime}$.

For addition $A_{2}$

$$
\begin{array}{rrrr}
r_{2}: & & \Rightarrow & q \\
s_{2}: & \neg q & \rightarrow & \neg q
\end{array}
$$

The only consequence of $D+A_{2}$ in $D L(\partial)$ related to $q$ and $\neg q$ is $-\Delta q$, and hence this is the only consequence of $D^{\prime}+A_{2}$ in $D L\left(\partial_{\|}\right)$related to $q$ and $\neg q$.

As we saw from $A_{1},(2.3 .1)$ or (2.3.2) holds for each rule $s$ for $\neg q$ in $D^{\prime}$. (2.3.2) cannot apply to $s_{2}$, by definition of modular addition. If (2.3.1) applies to $s_{2}$ in $D^{\prime}+A_{2}$ then $+\lambda \neg q \notin P_{\lambda}$ for $D^{\prime}+A_{2}$. Because neither $+\Delta q$ nor $+\Delta \neg q$ appear in $P_{\Delta}$ for $D^{\prime}+A_{2}$, this can only hold if every rule for $\neg q$ in $D^{\prime}+A_{2}$ contains a body literal $\alpha$ such that $+\lambda \alpha \notin P_{\lambda}$ for $D^{\prime}+A_{2}$, by the $+\lambda$ inference rule.

Now consider the application of the $+\partial_{\|}$inference rule to prove $+\partial_{\|} q$ in $D^{\prime}+A_{2}$. (2.1) is satisfied by $r_{2}$ and (2.2) is satisfied. For every rule for $\neg q$ in $D^{\prime}+A_{2},(2.3 .1)$ is satisfied, as shown in the previous paragraph. Hence $+\partial_{\|} q$ is a consequence of $D^{\prime}+A_{2}$. However, this contradicts the supposed simulation. Thus there is no $D^{\prime}$ that simulates $D$ wrt addition of rules in $D L\left(\partial_{\|}\right)$. 